\title{
THE ORIGIN OF DUST IN EARLY-TYPE GALAXIES AND IMPLICATIONS FOR ACCRETION ONTO SUPERMASSIVE BLACK HOLES
}

\author{
Paul Martini $^{1,4}$, Daniel Dicken ${ }^{2}$, and Thaisa Storchi-Bergmann ${ }^{3}$ \\ ${ }^{1}$ Department of Astronomy and Center for Cosmology and Astroparticle Physics, The Ohio State University, Columbus, OH 43210, USA \\ ${ }^{2}$ Institut de Astrophysique Spatiale, Paris, France \\ ${ }^{3}$ Instituto de Física, Universidade Federal do Rio Grande do Sul, Av. Bento Gonçalves 9500, Caixa Postal 15051, 91501-970 Porto Alegre, RS, Brazil \\ Received 2012 October 30; accepted 2013 February 16; published 2013 March 15
}

\begin{abstract}
We have conducted an archival Spitzer study of 38 early-type galaxies in order to determine the origin of the dust in approximately half of this population. Our sample galaxies generally have good wavelength coverage from $3.6 \mu \mathrm{m}$ to $160 \mu \mathrm{m}$, as well as visible-wavelength Hubble Space Telescope (HST) images. We use the Spitzer data to estimate dust masses, or establish upper limits, and find that all of the early-type galaxies with dust lanes in the HST data are detected in all of the Spitzer bands and have dust masses of $\sim 10^{5}-10^{6.5} M_{\odot}$, while galaxies without dust lanes are not detected at $70 \mu \mathrm{m}$ and $160 \mu \mathrm{m}$ and typically have $<10^{5} M_{\odot}$ of dust. The apparently dust-free galaxies do have $24 \mu \mathrm{m}$ emission that scales with the shorter-wavelength flux, yet substantially exceeds the expectations of photospheric emission by approximately a factor of three. We conclude this emission is dominated by hot, circumstellar dust around evolved stars that does not survive to form a substantial interstellar component. The order-of-magnitude variations in dust masses between galaxies with similar stellar populations rule out a substantial contribution from continual, internal production in spite of the clear evidence for circumstellar dust. We demonstrate that the interstellar dust is not due to purely external accretion, unless the product of the merger rate of dusty satellites and the dust lifetime is at least an order of magnitude higher than expected. We propose that dust in early-type galaxies is seeded by external accretion, yet the accreted dust is maintained by continued growth in externally accreted cold gas beyond the nominal lifetime of individual grains. The several Gyr depletion time of the cold gas is long enough to reconcile the fraction of dusty early-type galaxies with the merger rate of gas-rich satellites. As the majority of dusty early-type galaxies are also low-luminosity active galactic nuclei and likely fueled by this cold gas, their lifetime should similarly be several Gyr.
\end{abstract}

Key words: dust, extinction - galaxies: general - galaxies: ISM - infrared: galaxies - ISM: general

Online-only material: color figures

\section{INTRODUCTION}

One of the most striking characteristics of elliptical and lenticular galaxies is their apparent uniformity. Much more than their later-type cousins, early-type galaxies appear to have quite smooth and symmetric surface brightness profiles. Their morphological self-similarity is largely because most earlytype galaxies, and in particular ellipticals, are expected to be the result of nearly equal mass mergers that have produced symmetric stellar distributions via dynamical relaxation. This assembly process, combined with the generally large halos that host early-type galaxies, has also substantially heated the gas in their interstellar medium (ISM) such that cooling and subsequent star formation are quite inefficient. As a result, their stellar populations tend to be mostly old and well mixed, which reinforces their similar and smooth appearance.

Against the backdrop of these similarities, and the simplicity of this general picture, the differences between nominally similar early-type galaxies are cast into sharper relief than for the more highly structured late-type disk galaxy population. One striking difference is that some, but not all, early-type galaxies clearly contain cold, interstellar dust. This was revealed in IRAS observations that detected a number of elliptical galaxies at $100 \mu \mathrm{m}$ (Jura et al. 1987; Knapp et al. 1989; Goudfrooij \& de Jong 1995; Bregman et al. 1998). A study by Knapp et al. (1992) also demonstrated that ellipticals had emission at $10-12 \mu \mathrm{m}$ in excess of what was expected from stellar photospheres alone.

\footnotetext{
4 Visiting Astronomer, North American ALMA Science Center and University of Virginia, Charlottesville, VA 22903, USA.
}

This excess could be explained by circumstellar dust emission from evolved stars and proved to be a strong observational confirmation of expectations for mass loss from old stellar populations.

The presence of dust in early-type galaxies is interesting because it may provide additional information about their formation histories, the properties of dust when embedded within a substantially greater fraction of hot gas than is present in typical spirals, and the evolution of the ISM, in addition to stellar mass loss from evolved stars. Yet the presence of dust is also in apparent conflict with the expectation that the dust destruction timescale should be relatively short ( $\tau_{\text {dust }} \sim 2 \times 10^{4} \mathrm{yr}$ ), largely due to sputtering of small grains in their hot ISM (Draine \& Salpeter 1979b).

Observations with the Hubble Space Telescope (HST) revealed that dust lanes were common and probably present in the majority of early-type galaxies. These observations showed dust lanes in absorption within hundreds of parsecs to a $\mathrm{kpc}$ of the nucleus (van Dokkum \& Franx 1995). This study also noted that the incidence of dust is higher is radio galaxies than in radio-quiet galaxies, estimated that the mass of dust ranged from $10^{3}$ to $10^{7} M_{\odot}$, and that the distribution of the dust lanes suggests external accretion. Subsequent studies of atomic (Morganti et al. 2006) and molecular gas kinematics (Young et al. 2011; Davis et al. 2011) supported external accretion because the gas kinematics are often decoupled from the stellar kinematics.

While there is good, anecdotal evidence for external accretion, the evolved stars in early-type galaxies are also a potential source for the dust (Knapp et al. 1992). Athey et al. (2002) 
showed that the mid-infrared emission from early-type galaxies is consistent with stellar mass loss of order $0.1-1 M_{\odot} \mathrm{yr}^{-1}$ for a typical $L^{*}$ galaxy. For a dust-to-gas ratio of $\epsilon_{\mathrm{dg}}=0.005$ and a dust lifetime of $10^{7}-10^{8} \mathrm{yr}$ characteristic of the Milky Way, this would lead to a typical reservoir of $5 \times 10^{3}$ to $5 \times 10^{5} M_{\odot}$ (or $10-100 M_{\odot}$ for $\left.\tau_{\text {dust }}=2 \times 10^{4} \mathrm{yr}\right)$. While this dust would initially match the spatial and kinematic distribution of the evolved stellar population, Mathews \& Brighenti (2003) suggest that cooling in dust-rich gas could lead to clumping and settling into the central kiloparsec on a timescale of $10^{8}-10^{9} \mathrm{yr}$. While this substantially exceeds the expected lifetime of individual dust grains, the clumpiness may sufficiently shield the dust to produce a steady-state dust mass that is consistent with the observations. However, the internal origin hypothesis does not explain the dramatic variation in dust mass between otherwise quite similar galaxies. Temi et al. (2004) showed that there is no correlation between the far-infrared luminosity of early-type galaxies and their visible-wavelength luminosities, even though visible-wavelength light should correlate reasonably well with the number of mass-losing stars. This absence of a correlation can be more readily explained by the external accretion hypothesis.

Another important aspect of these studies was the observation that the majority of the early-type galaxies with dust also have emission-line nuclei that often indicate the presence of a LINER or Seyfert galaxy (van Dokkum \& Franx 1995; Ravindranath et al. 2001; Lauer et al. 2005). In order to investigate this correlation with a well-selected sample of early-type galaxies, in Simões Lopes et al. (2007) we studied archival HST images of a carefully matched sample of active and inactive early-type hosts and determined that all active early-type galaxies also have dust within hundreds of parsecs of their nuclei, while only $\sim 25 \%$ of inactive galaxies had evidence for dust. This study showed that $\sim 60 \%$ of the early-type galaxy population has interstellar dust. As we noted in Simões Lopes et al. (2007), the high incidence of dust in early-type galaxies presents a key challenge to the external origin hypothesis, namely that $\sim 60 \%$ of the early-type galaxy population must have accreted a gas and dust-rich dwarf within a time period comparable to the dust destruction time. For a dust destruction timescale of $10^{7}-10^{8} \mathrm{yr}$, this implies a very high rate of gas-rich mergers.

A missing ingredient in the Simões Lopes et al. (2007) study is that there was no constraint on the dust mass distribution in the early-type galaxies, which would provide a useful constraint on the mass range and consequently mass ratio of the satellite galaxies required to provide the observed mass of dust. Simões Lopes et al. (2007) also employed the structure map technique of Pogge \& Martini (2002) to identify dust lanes. As this is a contrast enhancement technique, it is insensitive to uniform or diffusely distributed dust. Uniformly distributed dust in the galaxies without dust lanes in the central kpc would provide strong support for the internal origin hypothesis.

Over the last few years, several studies with Spitzer and Herschel have assembled far-infrared measurements and estimated dust masses and basic dust properties for many early-type galaxies. One result of this work is that the dust in early-type galaxies is warmer than in spiral galaxies (Bendo et al. 2003; Skibba et al. 2011; Smith et al. 2012; Auld et al. 2013), which may be due to more intense radiation fields or different dust grain properties. The Herschel KINGFISH survey showed that early-type galaxies exhibit a strong correlation between the dust to stellar flux ratio and the specific star formation rate, which could be due to low levels of ongoing star formation (Skibba et al. 2011). The Herschel Reference Survey (Smith et al. 2012) and the Herschel Virgo Cluster Survey (Auld et al. 2013) detect many early-type galaxies and measure dust masses in the range of $\sim 10^{5}-10^{7} M_{\odot}$. The Smith et al. (2012) study concluded that much of this dust was acquired from interactions due to the wide range in dust to stellar mass ratio. A similar conclusion was reached by Rowlands et al. (2012), who detected $5.5 \%$ of luminous early-type galaxies in the Herschel-ATLAS/GAMA study. While only sensitive to quite high dust masses (their mean detected dust mass was $5.5 \times 10^{7} M_{\odot}$ ), they conclude that these high dust masses could not be produced internally.

In this paper, we present a Spitzer archival study of the origin of the dust in early-type galaxies. Our sample is composed of galaxies in the Simões Lopes et al. (2007) early-type galaxy sample that have both IRAC and MIPS observations. We describe this sample in Section 2 and our data processing and photometry in Section 3. All of the galaxies in this sample are detected in the IRAC bands, which are dominated by stellar emission, as well as in the $24 \mu \mathrm{m}$ MIPS band. Many are also detected in the longer-wavelength MIPS bands. The $24 \mu \mathrm{m}$ emission from galaxies not detected at longer wavelengths appears to be dominated by circumstellar dust. We describe how we account for this emission in Section 4. To derive the dust masses and properties of the dust, we fit these galaxies with the dust models of Draine \& Li (2007). These fits and the derived properties are described in Section 5. We use these data to develop a new hypothesis for the origin of the dust in early-type galaxies in Section 6 and summarize our results in Section 7.

\section{SAMPLE}

Our sample is composed of 38 bright, nearby, early-type galaxies (types E, E/S0, and S0). They constitute all of the early-type galaxies from the HST study of Simões Lopes et al. (2007) that have observations in at least two MIPS bands. The goal of the Simões Lopes et al. (2007) study was to compare dust structure in the central hundreds of parsecs between active and inactive galaxies and that sample contained equal numbers of well-matched active and inactive galaxies from the Palomar survey of Ho et al. (1995). The Palomar survey is very well suited for this purpose because it consists of very high signalto-noise ratio $(\mathrm{S} / \mathrm{N})$ spectroscopy of all bright galaxies in a fixed region of the sky. Furthermore, all of the nuclear spectra have been classified as either absorption-line nuclei, H II galaxies, LINERs, transition objects, or Seyfert galaxies (Ho et al. 1997b). The Palomar survey sample is thus both unbiased with respect to nuclear activity and has homogeneous and sensitive nuclear classifications. A further advantage is that there are a large number of ancillary data products, which are described in Ho (2008).

The Simões Lopes et al. (2007) study began with all of the LINERs and Seyfert galaxies (hereafter simply active galaxies) in the Palomar sample with HST observations and, for each active galaxy, identified a control (inactive) galaxy with the same morphology, distance, luminosity, and axis ratio. They identified 26 early-type pairs with relatively strict matching criteria (morphological $T$ type within 1 unit, absolute $B$ magnitude within $1 \mathrm{mag}$, distance within 50\%) and eight additional pairs of early-type galaxies that were not as well matched. These 34 pairs of active and inactive early-type galaxies had very similar distributions of distances, luminosities, and morphological types and are consequently very well suited to compare the differences between active and inactive early-type galaxies. As noted in the Introduction, that HST study found that all active, early-type 


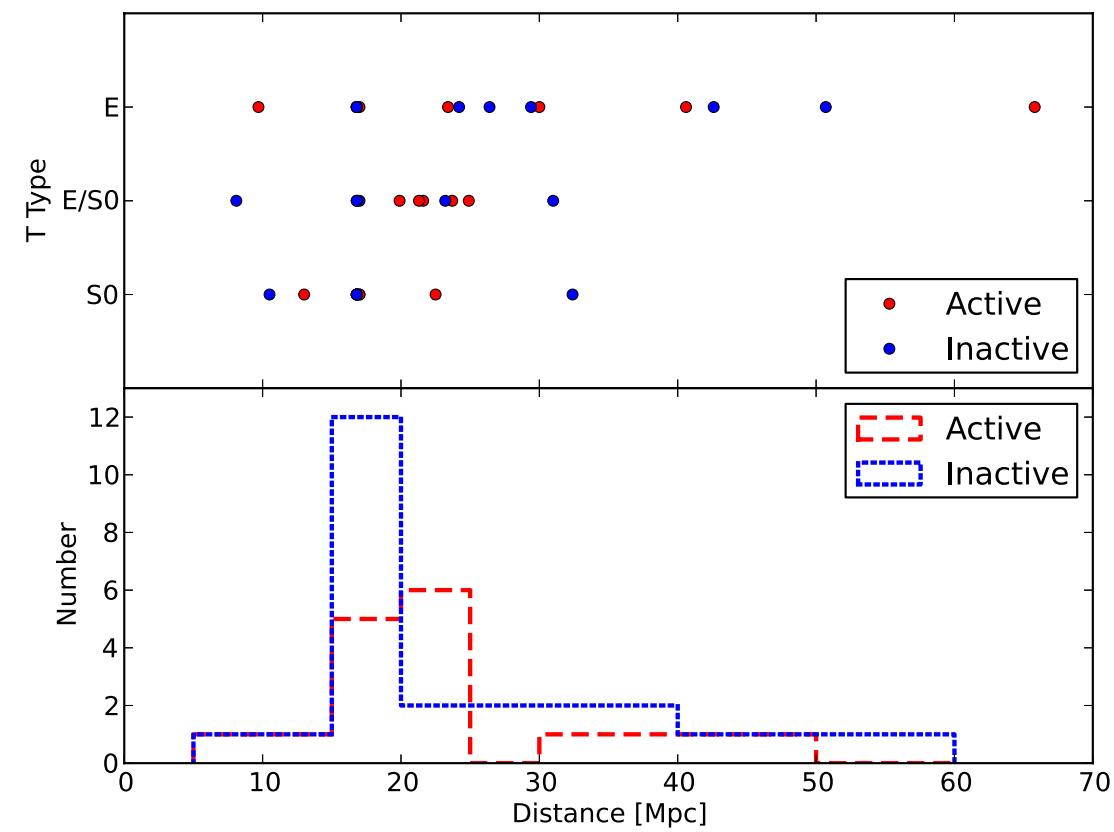

Figure 1. Sample distribution as a function of morphological type, activity, and distance. Top panel: active and inactive galaxies (red and blue circles, respectively) as a function of morphological type and distance. Bottom panel: histogram of all morphological types of the active and inactive galaxies (red and blue lines, respectively) as a function of distance. The substantial overdensity at $16.8 \mathrm{Mpc}$ is because that bin includes the Virgo Cluster.

(A color version of this figure is available in the online journal.)

galaxies possess some form of dust structure within several $100 \mathrm{pc}$ of their nuclei, whereas only $26 \%$ (nine) of the inactive galaxies exhibited evidence for such dust. These data thus show there is a strong dichotomy between dusty and active early-type galaxies and typically dust-free and inactive early-type galaxies. Furthermore, as Ho et al. (1997b) found that 50\% of all earlytype galaxies are classified as LINERs or Seyferts (active), this result also indicates that about $60 \%$ of all early-type galaxies have dust.

We constructed our sample from all of the early-type galaxies in the Simões Lopes et al. (2007) study that have observations in at least two MIPS bands, and in most cases all three are available. All of the galaxies also have IRAC observations, in addition to the HST observations. This sample has approximately equal numbers of active and inactive galaxies and should be an unbiased subset of the Simões Lopes et al. (2007) sample because these galaxies were targeted for MIPS observations by a multitude of small programs. Because of this selection, and a desire not to decrease the sample size further, we did not attempt to create a pair-matched sample from this subset. We did confirm that there are no obvious trends between the active and inactive subsets as a function of distance and morphological type. Figure 1 shows the distance distribution for galaxies classified as type E, E/SO, and SO. While the true ellipticals extend out to larger distances than the two other types, the distribution of active and inactive galaxies are similar. The morphological classification, activity type, and distance for all of the galaxies are presented in Table 1, along with whether or not they were observed to have dust structures in the study of Simões Lopes et al. (2007). Figure 2 presents HST images from that study, as well as Spitzer IRAC and MIPS images, for galaxies in this sample.

\section{DATA PROCESSING AND PHOTOMETRY}

We obtained all available IRAC and MIPS data for these galaxies from the Spitzer archive. The IRAC data correspond to images in bandpasses centered at approximately $3.6 \mu \mathrm{m}$, $4.5 \mu \mathrm{m}, 5.7 \mu \mathrm{m}$, and $8 \mu \mathrm{m}$. For most galaxies, the first three bands are dominated by stellar emission, while the fourth is dominated by emission from polycyclic aromatic hydrocarbons (PAHs). For most early-type galaxies, the fourth band is also dominated by stellar emission, rather than PAHs, as we discuss further below. The MIPS data correspond to images in bandpasses with nominal centers at $24 \mu \mathrm{m}, 70 \mu \mathrm{m}$, and $160 \mu \mathrm{m}$. Emission at $24 \mu \mathrm{m}$ is typically dominated by hot dust, which is either interstellar or circumstellar, while emission at longer wavelengths is dominated by cooler, diffuse, interstellar dust. We typically started our analysis with the basic calibrated data (BCD) for the MIPS observations and the post-BCD (PBCD) products for the IRAC observations. In the subsections below we describe any additional processing, photometric measurements, and how we determined upper limits.

\subsection{IRAC Data}

The PBCD data for these galaxies are sufficiently robust that no additional processing was necessary in most cases. The few exceptions were galaxies that had relatively few IRAC observations. In these cases we obtained the BCD data from the archive and processed them into mosaics with the MOPEX software package from the Spitzer Science Center.

We measured aperture photometry in all four IRAC bands with the phot task in IRAF. We typically adopted an aperture radius of $1^{\prime}$ because this includes nearly all of the observed dust emission at longer wavelengths. In some cases we used an 0.5 radius due to either nearby, bright objects or the extent of the imaging data in one or more bands. In several cases bright, foreground stars were in the aperture and we masked them out by replacing those pixel values with the median of nearby pixels. The aperture size and photometry for each galaxy is listed in Table 2. These measurements include the extended source aperture correction. 
Table 1

Sample Properties

\begin{tabular}{|c|c|c|c|c|}
\hline $\begin{array}{l}\text { Name } \\
\text { (1) }\end{array}$ & $\begin{array}{c}\text { Morph } \\
\text { (2) }\end{array}$ & $\begin{array}{c}\text { Activity } \\
\text { (3) }\end{array}$ & $\begin{array}{c}\text { Distance } \\
\text { (4) }\end{array}$ & $\begin{array}{c}\text { HST Dust? } \\
\text { (5) }\end{array}$ \\
\hline NGC 0315 & $\mathrm{E}$ & Active & 65.8 & $\mathrm{Y}$ \\
\hline NGC 0821 & E/S0 & Inactive & 23.2 & $\mathrm{~N}$ \\
\hline NGC 1023 & S0 & Inactive & 10.5 & $\mathrm{~N}$ \\
\hline NGC 2300 & E/S0 & Inactive & 31.0 & $\mathrm{~N}$ \\
\hline NGC 2768 & E/S0 & Active & 23.7 & $\mathrm{Y}$ \\
\hline NGC 2787 & So & Active & 13.0 & $\mathrm{Y}$ \\
\hline NGC 3226 & $\mathrm{E}$ & Active & 23.4 & $\mathrm{Y}$ \\
\hline NGC 3377 & E/S0 & Inactive & 8.1 & $\mathrm{Y}$ \\
\hline NGC 3414 & So & Active & 24.9 & $\mathrm{Y}$ \\
\hline NGC 3607 & S0 & Active & 19.9 & $\mathrm{Y}$ \\
\hline NGC 3640 & $\mathrm{E}$ & Inactive & 24.2 & $\mathrm{~N}$ \\
\hline NGC 3945 & S0 & Active & 22.5 & $\mathrm{Y}$ \\
\hline NGC 3998 & So & Active & 21.6 & $\mathrm{Y}$ \\
\hline NGC 4026 & S0 & Inactive & 17.0 & $\mathrm{Y}$ \\
\hline NGC 4138 & So & Active & 17.0 & $\mathrm{Y}$ \\
\hline NGC 4278 & $\mathrm{E}$ & Active & 9.7 & $\mathrm{Y}$ \\
\hline NGC 4291 & $\mathrm{E}$ & Inactive & 29.4 & $\mathrm{~N}$ \\
\hline NGC 4293 & SOa & Active & 17.0 & $\mathrm{Y}$ \\
\hline NGC 4365 & $\mathrm{E}$ & Inactive & 16.8 & $\mathrm{~N}$ \\
\hline NGC 4371 & S0 & Inactive & 16.8 & $\mathrm{Y}$ \\
\hline NGC 4382 & So & Inactive & 16.8 & $\mathrm{~N}$ \\
\hline NGC 4406 & $\mathrm{E}$ & Inactive & 16.8 & $\mathrm{~N}$ \\
\hline NGC 4526 & S0a & Inactive & 16.8 & $\mathrm{Y}$ \\
\hline NGC 4550 & So & Active & 16.8 & $\mathrm{Y}$ \\
\hline NGC 4570 & So & Inactive & 16.8 & $\mathrm{~N}$ \\
\hline NGC 4578 & S0 & Inactive & 16.8 & $\mathrm{~N}$ \\
\hline NGC 4589 & $\mathrm{E}$ & Active & 30.0 & $\mathrm{Y}$ \\
\hline NGC 4612 & So & Inactive & 16.8 & $\mathrm{~N}$ \\
\hline NGC 4621 & $\mathrm{E}$ & Inactive & 16.8 & $\mathrm{~N}$ \\
\hline NGC 4636 & $\mathrm{E}$ & Active & 17.0 & $\mathrm{Y}$ \\
\hline NGC 4649 & $\mathrm{E}$ & Inactive & 16.8 & $\mathrm{~N}$ \\
\hline NGC 4694 & $\mathrm{E}$ & Inactive & 16.8 & $\mathrm{Y}$ \\
\hline NGC 5077 & $\mathrm{E}$ & Active & 40.6 & $\mathrm{Y}$ \\
\hline NGC 5273 & So & Active & 21.3 & $\mathrm{Y}$ \\
\hline NGC 5308 & S0 & Inactive & 32.4 & $\mathrm{~N}$ \\
\hline NGC 5557 & $\mathrm{E}$ & Inactive & 42.6 & $\mathrm{~N}$ \\
\hline NGC 5576 & $\mathrm{E}$ & Inactive & 26.4 & $\mathrm{~N}$ \\
\hline NGC 7619 & $\mathrm{E}$ & Inactive & 50.7 & $\mathrm{~N}$ \\
\hline
\end{tabular}

Notes. Properties of the galaxies in the sample. Columns: (1) galaxy name; (2) morphological type; (3) activity type; (4) distance in Mpc; (5) presence or absence of dust in HST data from Simões Lopes et al. (2007). References for morphology, activity, and distance are provided in Simões Lopes et al. (2007).

The apertures are smaller than the full extent of the visible emission of these galaxies (e.g., $D_{25}$ ). While these smaller apertures have less noise, in some cases they may lead to underestimates of the total emission.

\subsection{MIPS Data}

The Spitzer MIPS data were processed further with the MOPEX package. In addition to the mosaicking and other processing (e.g., median filtering), we used the IDL program BCD column filter.pro to calculate and subtract the median value from each column for each BCD. This process significantly diminishes artifacts in the images with minimal loss of flux from the source.

The fluxes at $24 \mu \mathrm{m}$ and $70 \mu \mathrm{m}$ were measured with aperture photometry in the GAIA package and the same aperture sizes employed for the IRAC measurements. Aperture corrections were applied to these measurements. These corrections were derived from the point-spread function (PSF) of bright point sources in MIPS data. Aperture photometry proved impossible to use for the $160 \mu \mathrm{m}$ fluxes because it was difficult to accurately estimate the background. This is because the PSF at $160 \mu \mathrm{m}$ is comparable in size to the field of view in many cases. There is also the strong possibility of contamination by other sources. We therefore extracted the $160 \mu \mathrm{m}$ fluxes with the APEX package in MOPEX, which uses a PSF-fitting technique. The standard PSF available with the MOPEX package was used for this task.

We quantified several sources of measurement uncertainty. First, we calculated uncertainties for each galaxy in each band from the standard deviation of six measurements of the background. For some of the $70 \mu \mathrm{m}$ data and most of the $160 \mu \mathrm{m}$ data, this technique may not accurately capture the uncertainties as the field of view is too small. In these cases we set a lower limit on the uncertainty based on the noise in other, similar data sets with a larger field of view, such as work by Temi et al. (2009) and Bendo et al. (2012), as well as the confusion limit measurement by Frayer et al. (2009). Finally, we included the instrumental flux calibration uncertainties (of 4\%,10\%, and $12 \%$ for $24 \mu \mathrm{m}, 70 \mu \mathrm{m}$, and $160 \mu \mathrm{m}$, respectively). Calibration uncertainties dominate the uncertainties for the majority of the detections.

All of the galaxies were easily detected at $24 \mu \mathrm{m}$ and the apertures were centered on this emission. Detections at $70 \mu \mathrm{m}$ and $160 \mu \mathrm{m}$ were consistent with the same centroid as the $24 \mu \mathrm{m}$ emission. If there was not an obvious detection in one of these two bands, then an upper limit was measured at the coordinates of the $24 \mu \mathrm{m}$ emission. In all cases we quote $3 \sigma$ upper limits. Because of the size of our aperture, as well as PSF fitting at $160 \mu \mathrm{m}$, these upper limits are only valid if the dust is centrally concentrated.

Many of these archival data sets were included in previous studies, in particular Temi et al. (2009) and Bendo et al. (2012). Our fluxes generally agree to within the uncertainties with Temi et al. (2009), who employed a similar set of data processing and calibration techniques. The main differences are likely because Temi et al. (2009) employed apertures that were comparable in size to the visible extent of the galaxy. Our apertures are smaller, often by a factor of several, while at $160 \mu \mathrm{m}$ we employed a PSFbased measurement rather than aperture photometry. We have larger differences with the Bendo et al. (2012) measurements and the general sense of these differences is that our flux measurements tend to be smaller. Because Bendo et al. (2012) used similar aperture sizes to Temi et al. (2009), the difference is likely because Bendo et al. (2012) based their pipeline on an older set of processing and calibration tools. Two of the most significant differences we have traced to either the presence of a nearby companion (for NGC 3226) or clumps of emission at large radii (for NGC 4406, see also Gomez et al. 2010).

As our apertures are smaller than the full extent of the stellar emission (although not necessarily the dust emission), our measurements will underestimate the flux if the emission is more extended. In most cases, these differences appear to be at most on the order of a few tens of percent. The only significant difference is NGC 4526, where our $160 \mu \mathrm{m}$ measurement is only $60 \%$ of the value reported by Bendo et al. (2012), and about a factor of two below the PACS $160 \mu \mathrm{m}$ measurement of Auld et al. (2013), which has a much larger field of view and consequently better background measurement. We discuss the implications of our measurements on the inferred dust masses in Section 5.1.2. 
Table 2

Spitzer Photometry

\begin{tabular}{|c|c|c|c|c|c|c|c|c|}
\hline Name & Aperture & $3.55 \mu \mathrm{m}$ & $4.49 \mu \mathrm{m}$ & $5.73 \mu \mathrm{m}$ & $7.87 \mu \mathrm{m}$ & $23.68 \mu \mathrm{m}$ & $71.42 \mu \mathrm{m}$ & $155.9 \mu \mathrm{m}$ \\
\hline NGC 0315 & $1^{\prime}$ & 88.0 & 55.0 & 39.6 & 30.8 & $98.8(4.0)$ & $320.3(33.4)$ & $475(58)$ \\
\hline NGC 0821 & 0.5 & 100.9 & 60.6 & 40.2 & 25.1 & $8.8(0.7)$ & $<25.9$ & $<87.4$ \\
\hline NGC 1023 & $1^{\prime}$ & 330.0 & 197.8 & 135.9 & 82.7 & $52.3(2.1)$ & $<31.5$ & $<29.7$ \\
\hline NGC 2300 & $1^{\prime}$ & 122.6 & 73.0 & 49.4 & 30.2 & $19(0.8)$ & $<16.9$ & $<66.6$ \\
\hline NGC 2768 & $1^{\prime}$ & 136.1 & 83.8 & 58.4 & 39.6 & $32.4(1.3)$ & $513.2(51.5)$ & $486(61)$ \\
\hline NGC 2787 & $1^{\prime}$ & 178.4 & 107.8 & 78.6 & 60.8 & $40.5(1.6)$ & $962.9(96.6)$ & 833 (103) \\
\hline NGC 3226 & $0^{\prime} .5$ & 59.6 & 36.0 & 29.1 & 26.9 & $28(1.2)$ & $270.2(28.0)^{p}$ & $\ldots$ \\
\hline NGC 3377 & $1^{\prime}$ & 141.1 & 87.4 & 58.5 & 36.1 & $17.2(0.7)$ & $55.3(12.3)$ & $160(22)$ \\
\hline NGC 3414 & 0.5 & 98.8 & 59.9 & 42.1 & 30.5 & $26.2(1.1)$ & $272(28.5)$ & $\ldots$ \\
\hline NGC 3607 & $1^{\prime}$ & 0.0 & 123.5 & 0.0 & 105.4 & $89(3.6)$ & $1620.1(162.3)$ & $2314(278)$ \\
\hline NGC 3640 & 0.5 & 138.0 & 84.3 & 56.7 & 35.3 & $19.5(0.8)$ & $<25.6$ & $<49.8$ \\
\hline NGC 3945 & $1^{\prime}$ & 133.6 & 83.0 & 57.4 & 34.8 & $34.4(1.4)$ & $268.1(27.4)$ & $\ldots$ \\
\hline NGC 3998 & $1^{\prime}$ & 199.4 & 127.0 & 96.8 & 74.9 & $149.5(6.0)$ & $521.5(52.5)$ & $615(74)$ \\
\hline NGC 4026 & $1^{\prime}$ & 151.2 & 97.9 & 67.0 & 40.8 & $20.3(0.8)$ & $156(16.2)$ & $288(35)$ \\
\hline NGC 4138 & $1^{\prime}$ & 94.1 & 62.1 & 64.6 & 102.8 & $173.9(7.0)$ & $2121.2(212.2)$ & 3555 (427) \\
\hline NGC 4278 & $1^{\prime}$ & 201.9 & 122.6 & 86.4 & 62.3 & $46.3(1.9)$ & $709.1(71.4)$ & $741(90)$ \\
\hline NGC 4291 & $1^{\prime}$ & 84.3 & 51.9 & 34.4 & 20.6 & $21.4(0.9)$ & $<32.9$ & $<39.1$ \\
\hline NGC 4293 & $1^{\prime}$ & 78.9 & 61.5 & 72.5 & 114.0 & $528.9(21.2)$ & $5193(519.3)$ & $6890(827)$ \\
\hline NGC 4365 & $1^{\prime}$ & 191.7 & 116.0 & 81.0 & 49.6 & $31.2(1.3)$ & $<16.0$ & $<35.3$ \\
\hline NGC 4371 & $1^{\prime}$ & 107.0 & 63.8 & 43.1 & 27.3 & $15.1(0.6)$ & $30.5(6.3)$ & $<29.7$ \\
\hline NGC 4382 & $1^{\prime}$ & 206.8 & 131.2 & 94.9 & 58.8 & $54(2.2)$ & $<29.0$ & $<34.4$ \\
\hline NGC 4406 & $1^{\prime}$ & 206.3 & 122.5 & 83.8 & 51.2 & $40.1(1.6)$ & $<27.3$ & $<31.6$ \\
\hline NGC 4526 & $1^{\prime}$ & 310.6 & 191.7 & 170.0 & 224.2 & $284.8(11.4)$ & $7599.6(760.0)$ & $7049(846)$ \\
\hline NGC 4550 & 0.5 & 71.9 & 45.0 & 30.2 & 21.4 & $9.6(0.5)$ & $166.8(18.3)$ & $\ldots$ \\
\hline NGC 4570 & $1^{\prime}$ & 150.7 & 90.5 & 60.0 & 36.9 & $14.5(0.6)$ & $<25.0$ & $<29.7$ \\
\hline NGC 4578 & $1^{\prime}$ & 57.1 & 34.9 & 23.3 & 14.3 & $6.9(0.4)$ & $<29.0$ & $<29.7$ \\
\hline NGC 4589 & 0.5 & 103.9 & 64.4 & 44.8 & 29.9 & $14(0.6)$ & $204.8(20.9)$ & $326(40)$ \\
\hline NGC 4612 & $1^{\prime}$ & 62.3 & 38.7 & 25.8 & 16.4 & $9.6(0.5)$ & $<17.8$ & $<33.4$ \\
\hline NGC 4621 & $1^{\prime}$ & 224.6 & 132.1 & 89.8 & 55.5 & $33.2(1.3)$ & $<16.4$ & $<29.7$ \\
\hline NGC 4636 & $1^{\prime}$ & 167.2 & 99.4 & 68.2 & 42.4 & $24(1.0)$ & $112.8(16.0)$ & $\ldots$ \\
\hline NGC 4649 & 0.5 & 399.7 & 234.7 & 161.6 & 99.1 & $59.4(2.4)$ & $<18.7$ & $<29.7$ \\
\hline NGC 4694 & $1^{\prime}$ & 43.1 & 28.2 & 37.4 & 73.2 & $117.5(4.7)$ & $1391.9(139.3)$ & $2170(262)$ \\
\hline NGC 5077 & $1^{\prime}$ & 93.1 & 56.5 & 38.8 & 25.3 & $20.3(0.8)$ & $171.8(19.2)$ & $192(26)$ \\
\hline NGC 5273 & $1^{\prime}$ & 39.2 & 27.0 & 24.2 & 27.7 & $95.5(3.8)$ & $685(68.8)$ & $610(74)$ \\
\hline NGC 5308 & $1^{\prime}$ & 88.5 & 54.1 & 36.7 & 21.9 & $7.6(0.3)$ & $<26.3$ & $\ldots$ \\
\hline NGC 5557 & $1^{\prime}$ & 94.6 & 57.3 & 39.2 & 23.7 & $14.8(0.6)$ & $<13.5$ & $<33.4$ \\
\hline NGC 5576 & $1^{\prime}$ & 119.0 & 74.2 & 51.8 & 31.3 & $16.1(0.7)$ & $<19.4$ & $<32.5$ \\
\hline NGC 7619 & $1^{\prime}$ & 96.9 & 59.5 & 40.7 & 24.6 & $13.3(0.5)$ & $<18.2$ & $<29.7$ \\
\hline
\end{tabular}

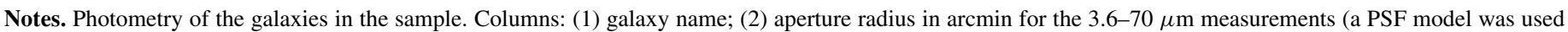

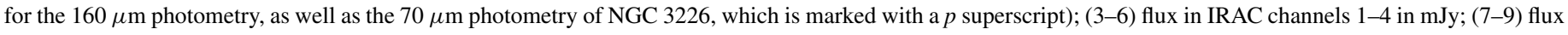

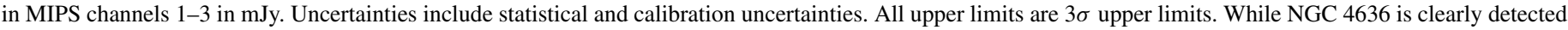
at $160 \mu \mathrm{m}$, we were unable to obtain a good photometric measurement.

\section{STELLAR AND CIRCUMSTELLAR EMISSION}

Most of the dust in galaxies is in the ISM. This dust is likely composed of a mix of amorphous silicate and graphitic grains, as well as small PAH particles. Both stars and PAH particles contribute emission to the $8 \mu \mathrm{m}$ IRAC band, so it is important to accurately determine the stellar contribution to the $8 \mu \mathrm{m}$ band in order to measure the PAH component. Similarly, both hot, circumstellar dust around evolved stars and hot, interstellar dust contribute emission to the $24 \mu \mathrm{m}$ MIPS band, so it is important to determine the circumstellar contribution to the $24 \mu \mathrm{m}$ band in order to quantify the warm component of the diffuse ISM. In the next two subsections, we describe how we used our sample of galaxies with no evidence for interstellar dust to obtain an empirical measurement of the stellar and circumstellar emission in the $8 \mu \mathrm{m}$ and $24 \mu \mathrm{m}$ bands.

\subsection{Stellar Contribution}

IRAC $8 \mu \mathrm{m}$ observations of most galaxies include contributions from both stellar photospheres and PAH molecules. In order to study the total flux and spatial distribution of PAHs, the conventional practice is to remove the stellar contribution by subtraction of a scaled version of the $3.6 \mu \mathrm{m}$ flux, where this scale factor is calculated from models of stellar photospheres. Helou et al. (2004) estimated that this value is 0.232 based on stellar population models from Starburst 99 (Leitherer et al. 1999) and noted this factor is not very sensitive to star formation history or metallicity. They do note that the $3.6 \mu \mathrm{m}$ emission may include a hot dust component, based on earlier work (Bernard et al. 1994; Hunt et al. 2002), but estimate that this is a small contribution. Draine et al. (2007) obtained a similar value of 0.260 with the assumption that the emission is described by a $5000 \mathrm{~K}$ blackbody.

The stellar contribution at $8 \mu \mathrm{m}$ is a small fraction of the total emission from the late-type spiral NGC 300 studied by Helou et al. (2004). This is also true for most of the galaxies in the SINGS sample studied by Draine et al. (2007). While approximately half of the galaxies in our sample are similarly expected to have substantial dust emission at $8 \mu \mathrm{m}$, others may have little to no dust emission in this band. For these galaxies, 

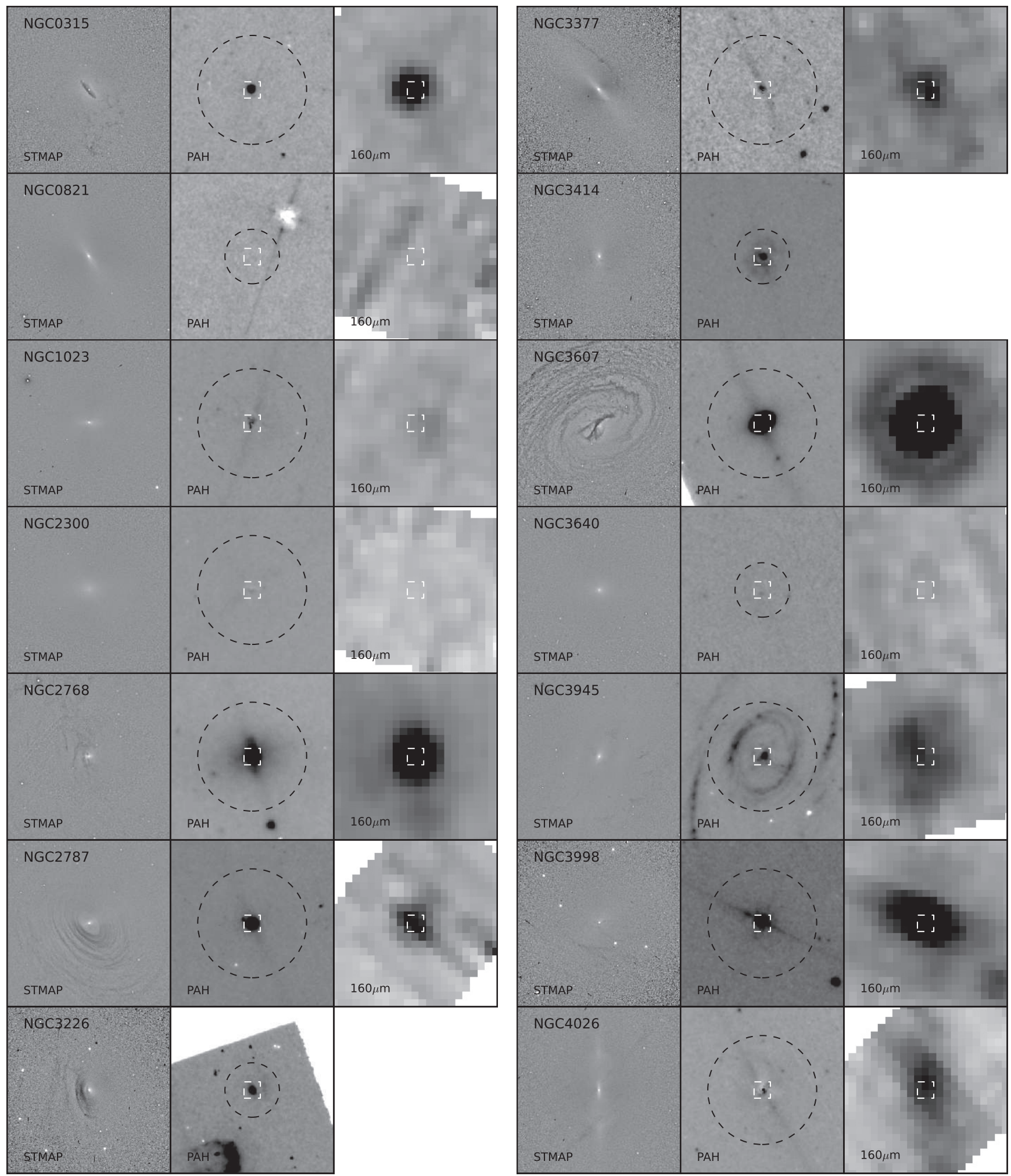

Figure 2. Structure maps (left), PAH images (middle), and MIPS $160 \mu \mathrm{m}$ images (right) for the sample. The structure map is $18^{\prime \prime}$ on a side, while the PAH and MIPS images are $180^{\prime \prime}$ on a side. The small box on the PAH and MIPS images indicates the field of view of the structure map. The black, dashed circle on the PAH image indicates the photometric aperture used for the 3.6-70 $\mu \mathrm{m}$ photometry (a PSF model was used for the $160 \mu \mathrm{m}$ data). The third panel is empty if $160 \mu \mathrm{m}$ MIPS data were unavailable for that galaxy.

an accurate estimate of the scale factor specific to early-type galaxy stellar populations is necessary to correctly identify and measure PAH emission at $8 \mu \mathrm{m}$.

We have empirically estimated the appropriate scale factor for early-type galaxies with the nominally dust-free galaxies in our sample. Figure 3 shows the ratios of $f_{8} / f_{3.6}$ and $f_{8} / f_{4.5}$ for the subset of the sample with the smallest values of these ratios as a function of $4.5 \mu \mathrm{m}$ luminosity. In both cases, all of the dust-free galaxies have similar values of these ratios, which correspond to $f_{8} / f_{3.6}=0.25 \pm 0.01$ and $f_{8} / f_{4.5}=0.42 \pm 0.01$. 

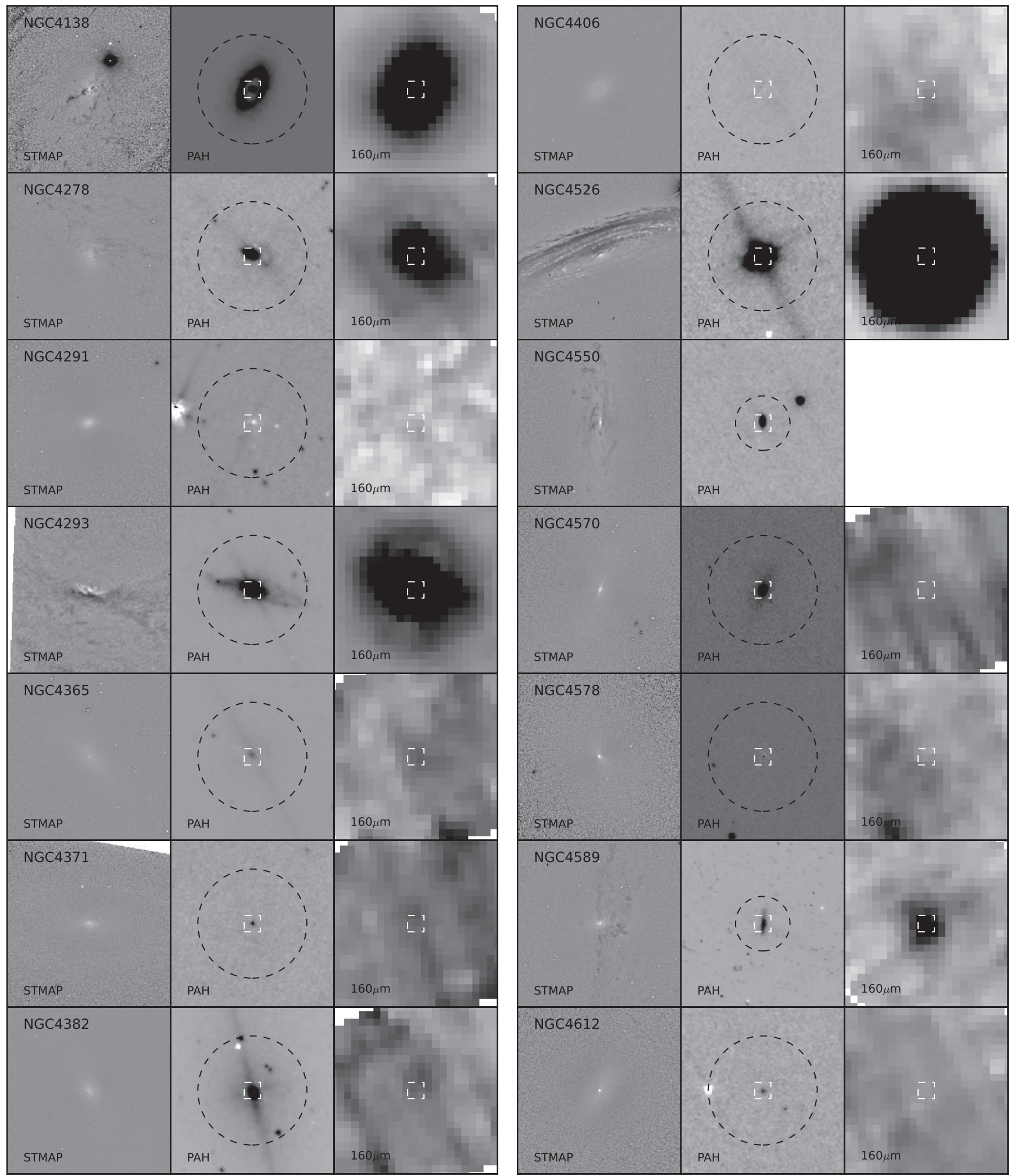

Figure 2. (Continued)

The uncertainty quoted for both ratios represents the rms variation of the dust-free galaxies. The average photometric uncertainty for the ratio measurement is also shown in each panel, although because it includes (correlated) absolute calibration uncertainties it is an overestimate of the true uncertainty in the individual points. The measured value for $f_{8} / f_{3.6}$ is consistent at $1 \sigma$ with the value of 0.260 chosen by Draine et al. (2007) and is $2 \sigma$ from the value of 0.232 calculated by Helou et al. (2004).

We have compared these values to the predicted flux ratios from stellar population models by Maraston (2005) and Conroy et al. (2009) to judge how well the models fit these flux ratios, examine the expected variation due to stellar population differences, and estimate the significance of the $24 \mu \mathrm{m}$ detections 

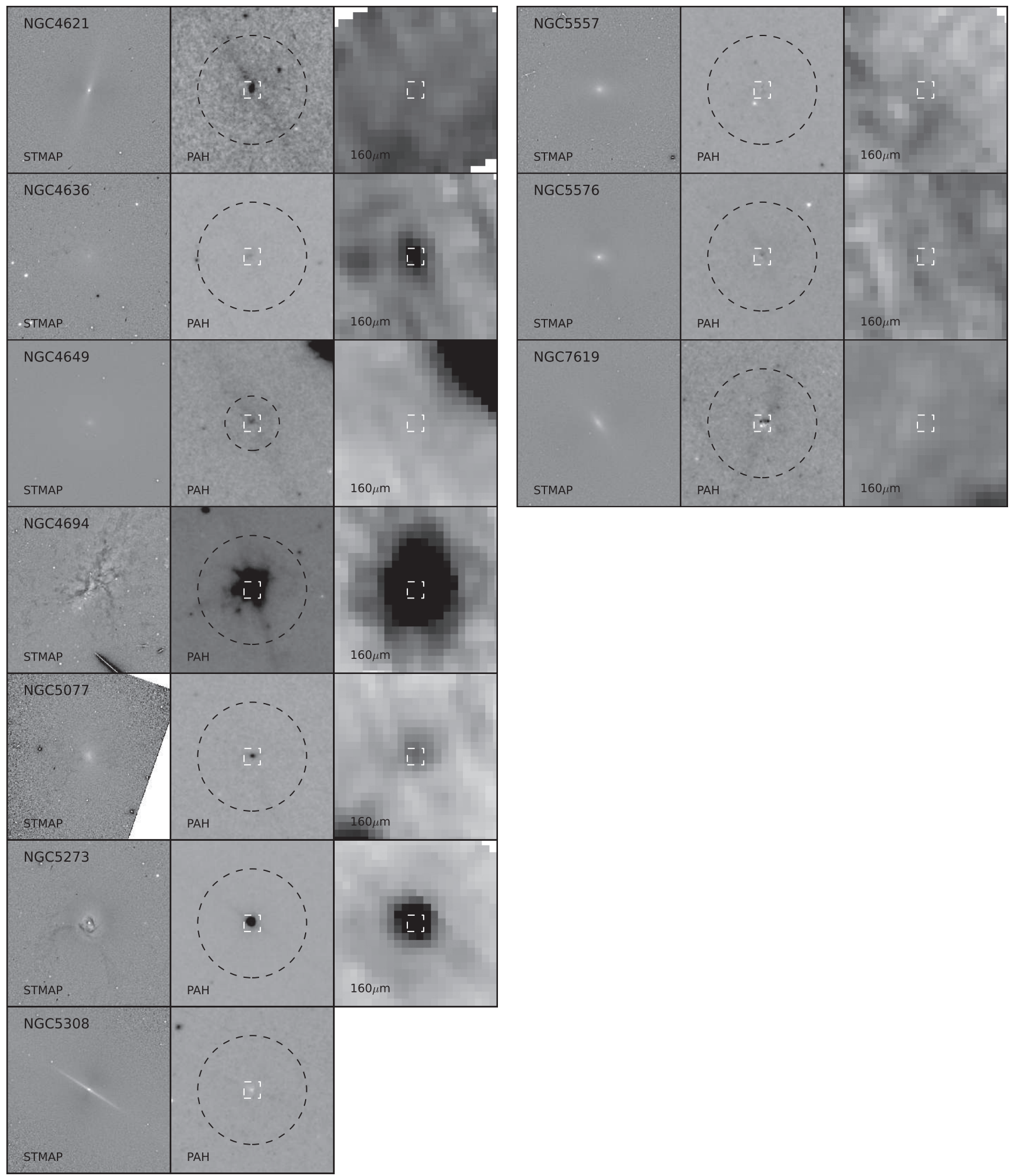

Figure 2. (Continued)

of apparently dust-free galaxies (in Section 4.2). As early-type galaxies are primarily composed of old stellar populations, and this component is expected to dominate the stellar emission in the infrared, we only examine models with single stellar population ages, but consider metallicities from solar to supersolar. From Maraston (2005) we calculate the $f_{8} / f_{3.6}$ and $f_{8} / f_{4.5}$ ratios for metallicities of $Z=0.02, Z=0.04$, and $Z=0.07$, a Kroupa initial mass function (IMF), and their red horizontalbranch prescription. From Conroy et al. (2009) we calculate these ratios for $Z=0.019$ and $Z=0.035$, a Kroupa IMF, the Padova isochrones, and the Basel library.

The flux ratios for these models as a function of population age are shown in Figure 4. The $f_{8} / f_{3.6}$ and $f_{8} / f_{4.5}$ ratios from the models agree well with the empirical data shown in Figure 3. 


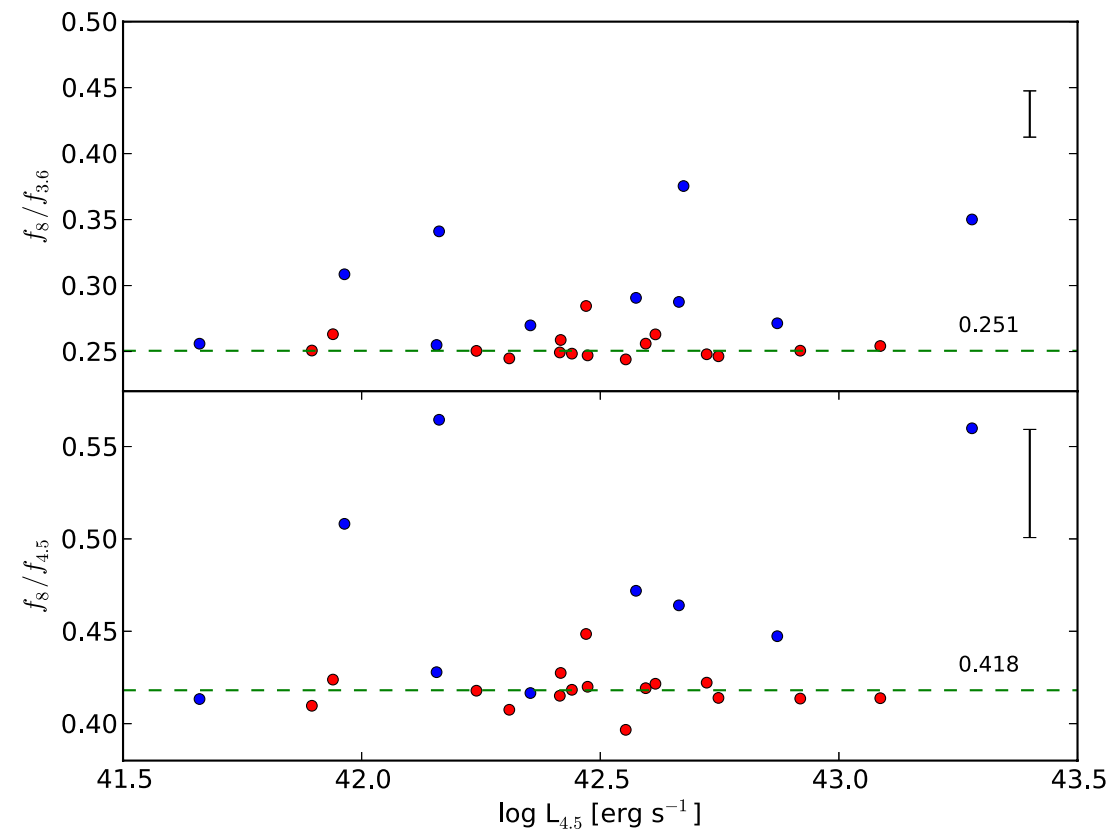

Figure 3. Ratios of $8 \mu \mathrm{m}$ to $3.6 \mu \mathrm{m}$ flux (top panel) and $8 \mu \mathrm{m}$ to $4.5 \mu \mathrm{m}$ flux (bottom panel) as a function of $4.5 \mu \mathrm{m}$ luminosity for galaxies with the smallest value of this ratio. Red points correspond to galaxies without evidence for dust, both based on upper limits at $160 \mu \mathrm{m}$ and the absence of dust structure in $H S T$ imaging. Blue points correspond to galaxies with $160 \mu \mathrm{m}$ detections and dust structure in $H S T$ imaging. The galaxies with no evidence for dust have median ratios of $f_{8} / f_{4.5}=0.418 \pm 0.011$ and $f_{8} / f_{3.6}=0.251 \pm 0.010$ and we adopt these values to remove the stellar contribution at $8 \mu \mathrm{m}$ for our early-type galaxy sample. The error bar in each panel represents the average uncertainty in the measurement of the ratio. Several galaxies with detections at $160 \mu \mathrm{m}$ (blue points) have similar value of this ratio, although some are substantially higher and off the upper end of the range shown on this figure.

(A color version of this figure is available in the online journal.)
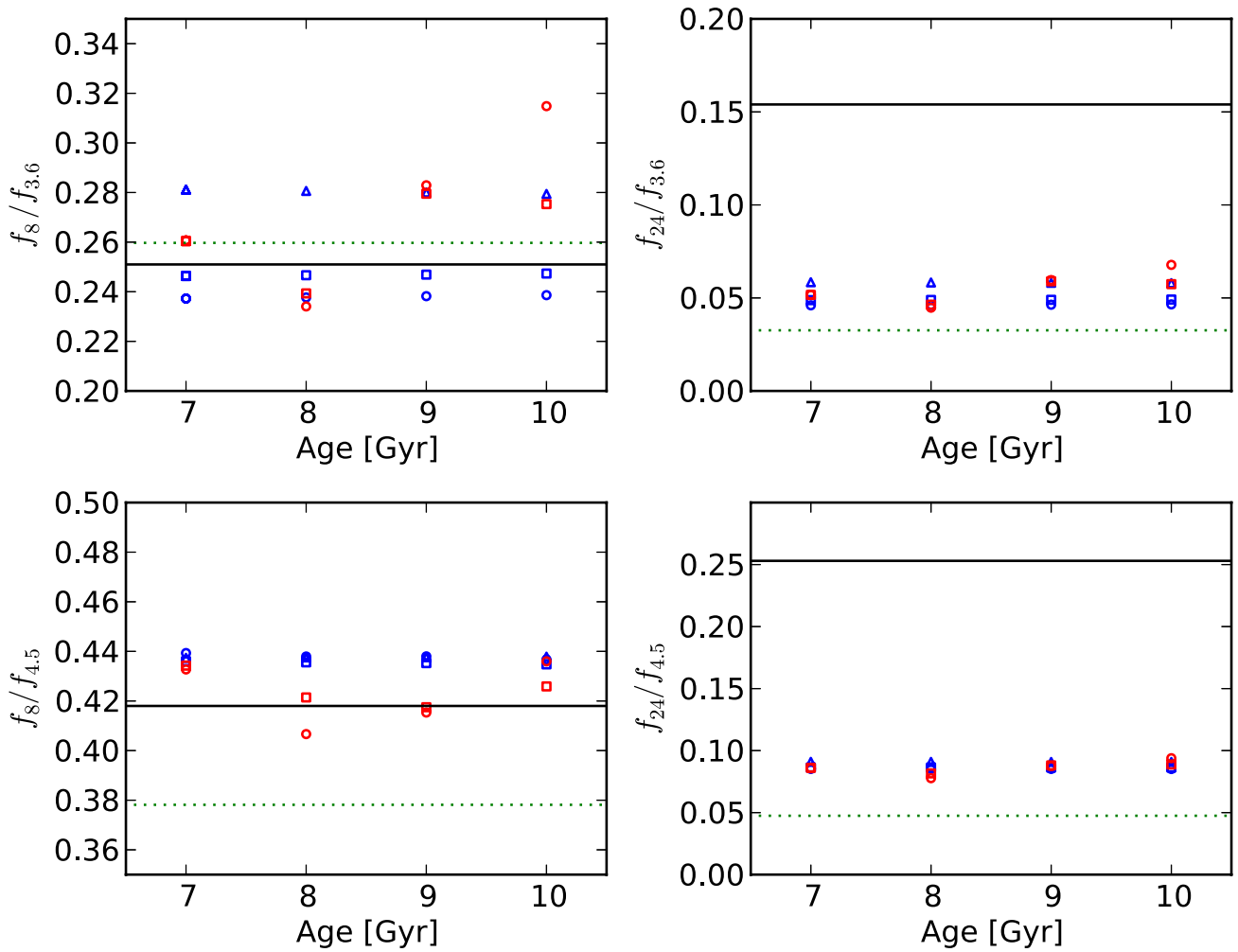

Figure 4. Spitzer IRAC and MIPS band flux ratios for stellar emission from various old stellar population models as a function of age (i.e., with no ISM or circumstellar dust contribution). The flux ratios shown are $f_{8} / f_{3.6}$ (top left), $f_{24} / f_{3.6}$ (top right), $f_{8} / f_{4.5}$ (bottom left), and $f_{24} / f_{4.5}$ (bottom right). The model points from Maraston (2005) are for $Z=0.02$ (blue circles), $Z=0.04$ (blue squares), and $Z=0.07$ (blue triangles). The model points from Conroy et al. (2009) are for $Z=0.019$ (red circles) and $Z=0.035$ (red squares). The empirical ratios (black solid line) and a $5000 \mathrm{~K}$ blackbody (dotted line) are also shown. The models are a reasonable representation of the IRAC flux ratios, but not the MIPS to IRAC flux ratios, which is evidence for a hot, circumstellar dust component. See Sections 4.1 and 4.2 for details.

(A color version of this figure is available in the online journal.) 


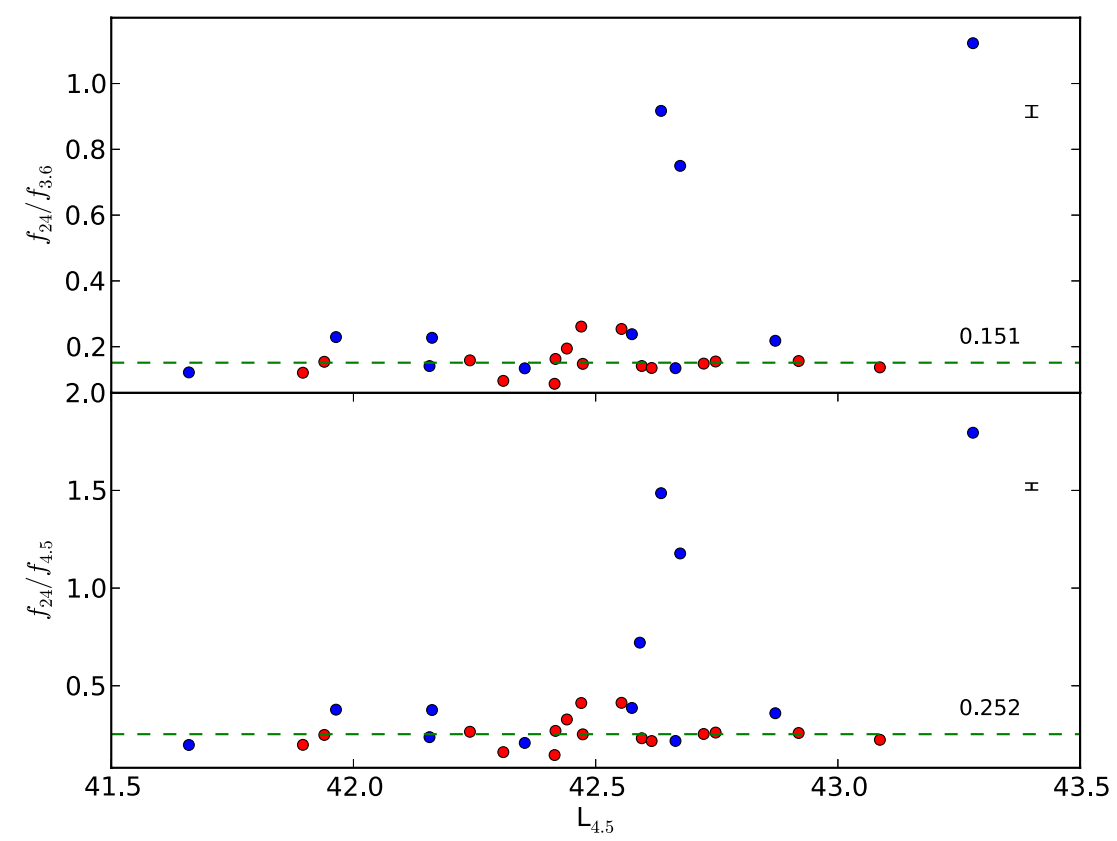

Figure 5. Same as Figure 3 for the ratios of $24 \mu \mathrm{m}$ to $3.6 \mu \mathrm{m}$ (top panel) and to $4.5 \mu \mathrm{m}$ (bottom panel). Red points correspond to galaxies without evidence for dust, both based on upper limits at $160 \mu \mathrm{m}$ and the absence of dust structure in $H S T$ imaging. Blue points correspond to galaxies with $160 \mu \mathrm{m}$ detections and dust structure in HST imaging. The galaxies with no evidence for dust have median ratios of $f_{24} / f_{4.5}=0.252 \pm 0.072$ and $f_{24} / f_{3.6}=0.151 \pm 0.045$. The error bar in each panel represents the average uncertainty in the measurement of the ratio.

(A color version of this figure is available in the online journal.)

The Maraston (2005) models indicate a trend of larger $f_{8} / f_{3.6}$ ratio at larger metallicities. The Conroy et al. (2009) models appear relatively insensitive to metallicity, but do show some increase at larger ages. The $f_{8} / f_{4.5}$ ratios for both sets of models agree well with each other and the data, and also appear essentially independent of age and metallicity. Models with different IMF prescriptions lead to essentially identical results and are not shown.

\subsection{Circumstellar Dust}

While the galaxies without evidence for dust in HST images were also undetected at $70 \mu \mathrm{m}$ and $160 \mu \mathrm{m}$, all are detected at $24 \mu \mathrm{m}$. Figure 5 displays a similar plot to Figure 3, but for $24 \mu \mathrm{m}$ rather than $8 \mu \mathrm{m}$. This figure indicates that the $24 \mu \mathrm{m}$ emission is relatively constant for the apparently dustfree galaxies, although the scatter is larger than for the $8 \mu \mathrm{m}$ emission. The larger scatter may be due to stellar population differences, such as age or metallicity. This was indicated by the work of Athey et al. (2002), who measured significant intrinsic scatter between the mid-infrared and $B$-band luminosities of nine early-type galaxies. The ratios and the rms variation we measure are $f_{24} / f_{4.5}=0.253 \pm 0.074$ and $f_{24} / f_{3.6}=0.154 \pm 0.047$. While some of the dusty galaxies also have consistent values of these ratios, the ratios for many are much larger and some are not shown on the figure.

These ratios for all galaxies, including the apparently dustfree galaxies, are several times larger than expected from stellar photospheric emission alone. The right panels of Figure 4 show the empirical $f_{24} / f_{3.6}$ and $f_{24} / f_{4.5}$ ratios compared to the same Maraston (2005) and Conroy et al. (2009) models described in the previous section. In both cases the empirical values are approximately a factor of three higher than all of the models. The models are also in good agreement with each other and show little evidence for significant variation as a function of stellar population age or metallicity. We therefore conclude that the $24 \mu \mathrm{m}$ emission is clearly in excess of stellar photospheric emission, yet it does scale with the flux of the stellar population.

The most likely origin of this excess $24 \mu \mathrm{m}$ flux is emission from hot dust in the circumstellar envelopes of evolved stars. For a population of galaxies with relatively uniform and old stellar populations, the fraction of evolved stars with circumstellar dust should be an approximately fixed fraction of the stellar mass, which in turn is reasonably well represented by the flux at $3.6 \mu \mathrm{m}$ and $4.5 \mu \mathrm{m}$. Models of infrared emission from dust shells around asymptotic giant branch (AGB) stars by Piovan et al. (2003) include substantial circumstellar emission from 10 to $40 \mu \mathrm{m}$ from old stars. This emission includes a local maximum that approximately falls within the $24 \mu \mathrm{m}$ band and has been identified with silicate emission in oxygen-rich AGB stars (Suh 2002). Bressan et al. (2007) studied Spitzer IRS spectroscopy of many early-type galaxies, including a number of the inactive galaxies in our sample (NGC 4365, NGC 4371, NGC 4382, NGC 4570, NGC 4621), and these data show clear evidence for dust emission beyond $8 \mu \mathrm{m}$ that is well fit by a dusty silicate circumstellar envelope model (Bressan et al. 1998). This emission spectrum is also well fit by a scaled version of Spitzer IRS data for stars in the globular cluster 47 Tuc studied by Lebzelter et al. (2006).

For galaxies with a substantial amount of cold, interstellar dust $\left(\geqslant 10^{6} M_{\odot}\right)$, such as late-type galaxies, the circumstellar dust emission at $24 \mu \mathrm{m}$ is relatively negligible. However, most of the galaxies in our sample are substantially more dust poor for their stellar mass. It is consequently important to estimate and subtract the circumstellar contribution to the $24 \mu \mathrm{m}$ MIPS photometry to obtain an accurate estimate, or upper limit, for the cold dust component. In the next section, we describe our dust model fits and how we incorporate the circumstellar dust emission into the interstellar dust mass estimates and upper limits. 


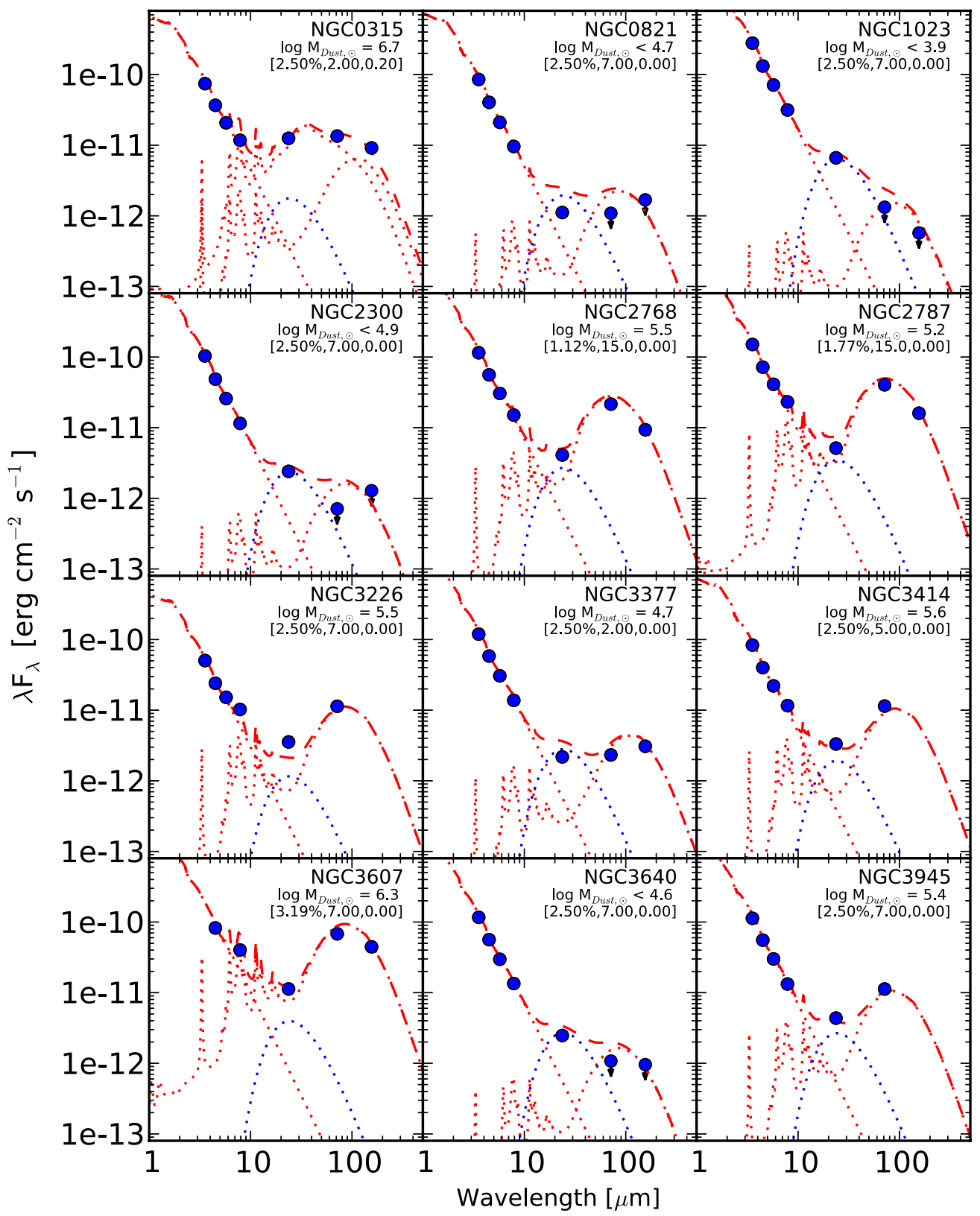

Figure 6. Infrared spectral energy distributions and model fits. The IRAC and MIPS photometric measurements (blue circles or arrows) are shown with a stellar model from Maraston (2005), diffuse and PDR dust models from Draine \& Li (2007) (red, dotted lines), and a simple circumstellar dust model (blue, dotted lines) that corresponds to a fixed fraction of the $4.5 \mu \mathrm{m}$ emission. The sum of these components is also shown (red, dashed line). The dust mass estimates, as well as either the best-fit or assumed dust model parameters $\left(q_{\mathrm{PAH}}, U_{\mathrm{min}}\right.$, and $\gamma$ ), are listed below each galaxy's name. The dust model fits are described in Section 5.1 .

(A color version of this figure is available in the online journal.)

\section{INTERSTELLAR DUST}

Approximately half of these early-type galaxies are detected in the MIPS $70 \mu \mathrm{m}$ and $160 \mu \mathrm{m}$ bands, which indicates the presence of cold, interstellar dust. In this section, we describe how we fit our IRAC and MIPS photometry for these galaxies with the dust models of Draine \& Li (2007), estimate the total dust mass, and compare the properties of the dust in early-type galaxies to dust in other morphological types. We also derive upper limits to the cold dust mass for galaxies that we do not detect at $70 \mu \mathrm{m}$ and $160 \mu \mathrm{m}$. The SEDs and our best fits are shown in Figure 6.

All of the galaxies with dust lanes in the HST structure maps are also detected at $8 \mu \mathrm{m}$. We use the empirical scale factor derived in Section 4.1 to measure the PAH emission in this bandpass and examine the spatial distribution of the PAHs. We also estimate the fraction of the dust in PAHs with the Draine \& $\mathrm{Li}$ (2007) models for the galaxies with suitable data and compare them to other studies.

\subsection{Fit to Draine \& Li Models}

Most of the dust in galaxies is in the ISM. This dust absorbs energy from starlight, which then re-emits this radiation at midand far-infrared wavelengths. The emission spectrum of this dust can be used to estimate the total mass in dust, as well as other properties, given a model for the typical grain size distribution and composition. These models are used to characterize the absorption and re-emission of the interstellar radiation field 


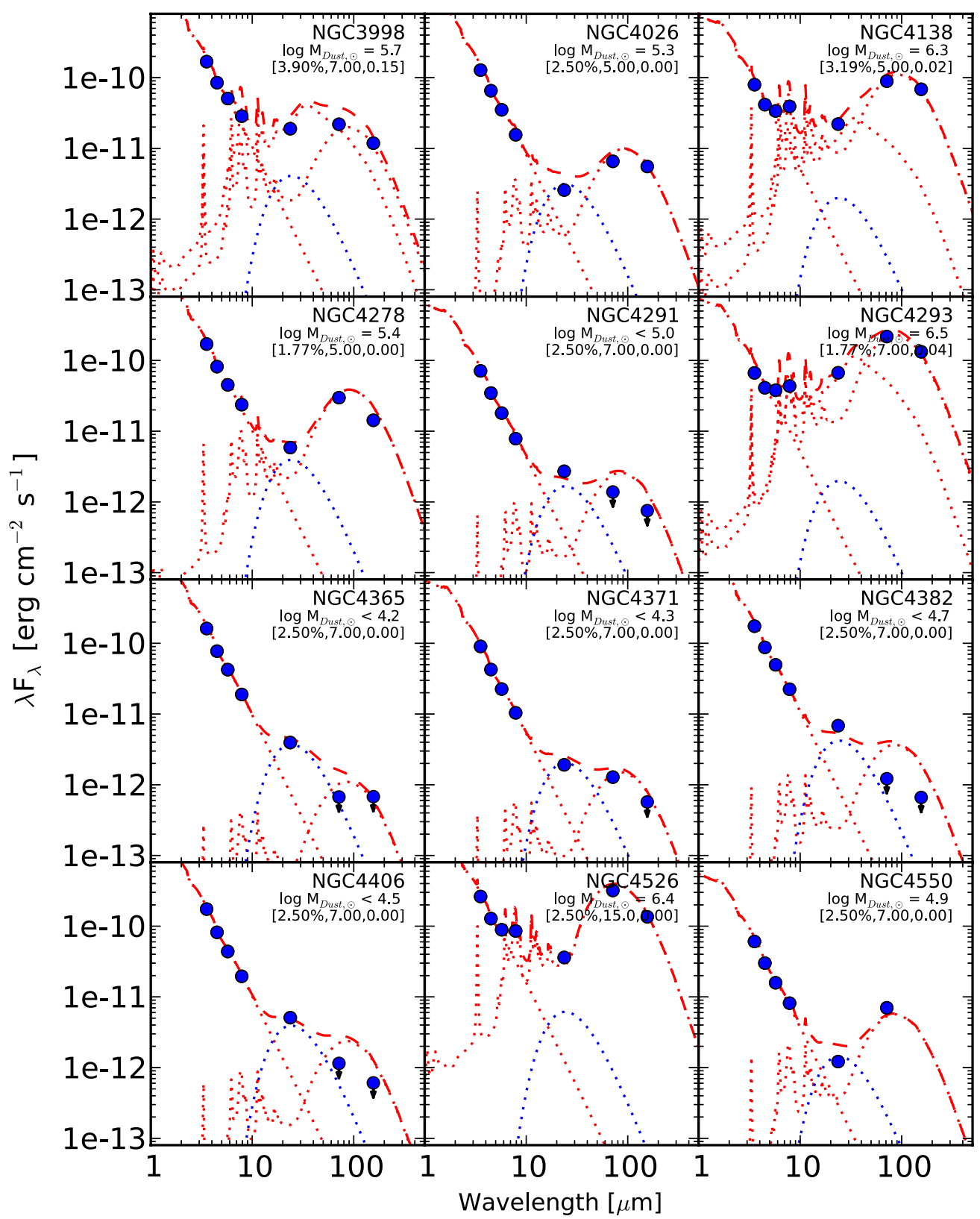

Figure 6. (Continued)

(ISRF) as a function of wavelength and may consequently be used to derive the dust mass from the observed luminosity.

We employ the models of Draine \& Li (2007) to characterize the properties of the dust in these galaxies and derive dust masses. These models assume that dust is comprised of a mixture of carbonaceous and silicate grains, and varying amounts of PAH particles. The main observational constraints on the grain size distribution, relative mixture and opacities of carbonaceous and silicate grains, and the properties of the PAH particles are the wavelength-dependent extinction observed in the Milky Way (Mathis et al. 1983), long-wavelength observations of the Milky Way (Finkbeiner et al. 1999), and studies of the properties of PAH emission from the Milky Way and nearby galaxies (e.g., Smith et al. 2007).

The mid- to far-infrared emission from dust grains depends on the incident ISRF, and thus a characterization of the distribution of starlight intensities is also necessary to infer the properties of the dust. Integrated measurements of entire galaxies will typically include emission from dust that has been heated by a wide range of different values of the local ISRF. While some studies perform detailed radiative transfer calculations to estimate the local value of the ISRF for individual dust grains (e.g., Popescu et al. 2000; Gordon et al. 2001; Piovan et al. 2006), this approach typically relies on many assumptions and may not be necessary to obtain reasonable estimates of the dust mass and other dust properties. Draine \& Li (2007) propose a simple parameterization of the ISRF where a fraction $\gamma$ of the dust mass is exposed to high-intensity radiation, such as may occur near the sites of star formation, and the remainder is exposed to the average ISRF. The dust exposed to a wide range of ISRF intensities, which Draine \& Li (2007) refer to as dust associated with photodissociation regions (or simply PDR dust) is exposed to a power-law distribution of intensities from $U_{\min }$ to $U_{\max }$, where $U$ is a dimensionless scale factor such that $U=$ 1 for the local ISRF derived by Mathis et al. (1983), $U_{\min }$ is the minimum value of the ISRF, $U_{\max }$ is the maximum value, and 


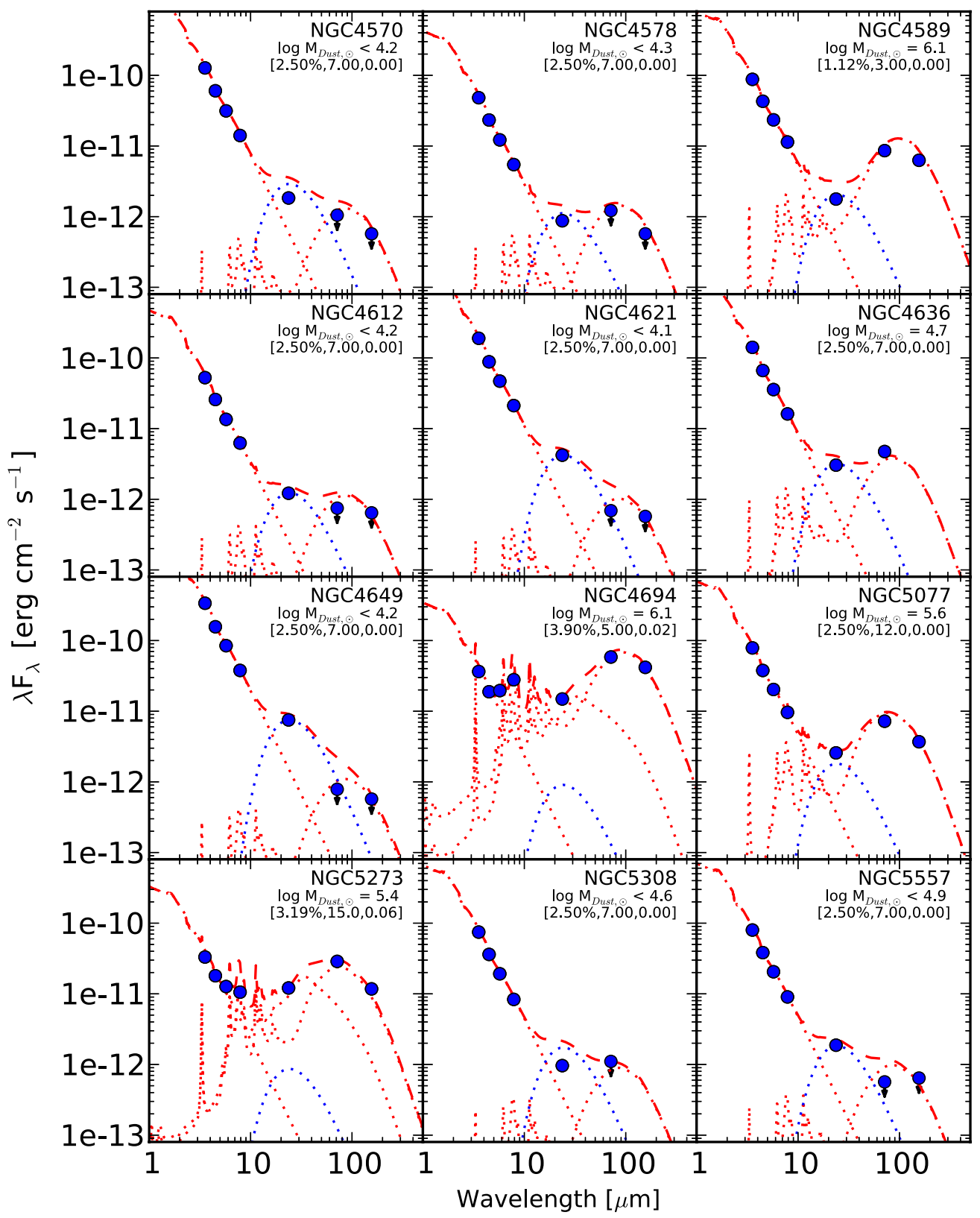

Figure 6. (Continued)

the power-law index is $\alpha$. The dust exposed to the average ISRF is exposed to a radiation field of intensity $U_{\min }$ and is referred to as diffuse dust. The diffuse dust is expected to be relatively cold and dominate the emission in the $70 \mu \mathrm{m}$ and $160 \mu \mathrm{m}$ bands, while if present the PDR dust may be important at shorter wavelengths, most notably the $24 \mu \mathrm{m}$ MIPS band. Draine et al. (2007) find that the spectral energy distributions (SEDs) of galaxies in the SINGS sample are satisfactorily reproduced with $U_{\max }=10^{6}$ and a power-law index of $\alpha=2$. We fix these two model parameters at those values for this study as well. The ISRF for the dust models is consequently just characterized by $U_{\min }$ and $\gamma$. As early-type galaxies have relatively little star formation, we do not expect large values of $\gamma$; however, $\gamma$ may not be completely negligible as some early-type galaxies have modest amounts of star formation. The PDR component may also represent dust near the active nucleus in the small number of moderate-luminosity active galactic nuclei (AGNs) in this sample.

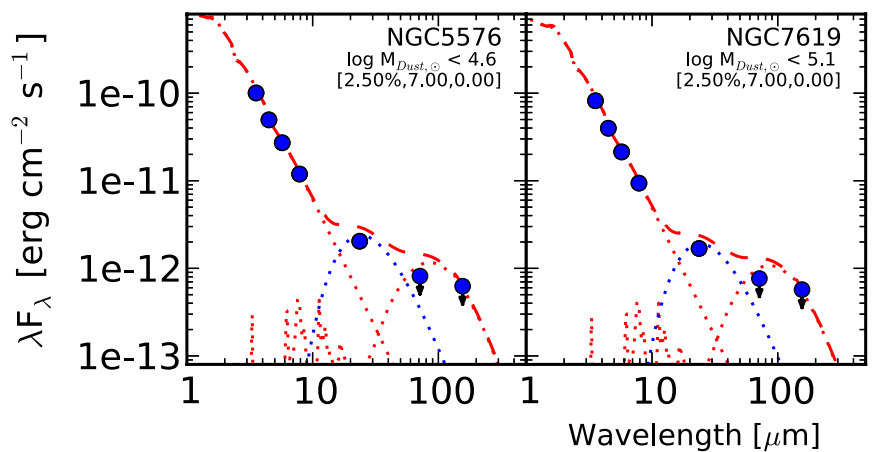

Figure 6. (Continued)

Very small dust grains and PAHs are subject to single-photon heating to high temperatures and thus emit at much shorter wavelengths than larger grains (Sellgren 1984). PAHs are also very likely to be the cause of the emission features observed 
at 3.3, 6.2, 7.7, 8.6, and $11.3 \mu \mathrm{m}$ in the Milky Way and most galaxies, and in particular the $7.7 \mu \mathrm{m}$ feature dominates the nonstellar emission in the IRAC $8 \mu \mathrm{m}$ band. This emission has been identified with $\mathrm{C}-\mathrm{C}$ stretch and $\mathrm{C}-\mathrm{H}$ bending modes, primarily from ionized PAHs. The Draine \& Li (2007) models include PAHs as small as 20 carbon atoms and note that PAHs with $<1000$ carbon atoms dominate the emission at wavelengths $\lambda<20 \mu \mathrm{m}$. They present seven different Milky Way dust models that have different size distributions of very small grains and different $\mathrm{PAH}$ fractions $q_{\mathrm{PAH}}$ of $0.5 \%-4.6 \%$, where $q_{\text {PAH }}=4.6 \%$ is a reasonable match to the Milky Way. Because each of these seven dust models has a different PAH fraction, $q_{\text {PAH }}$ uniquely describes the dust model. The dust emission from a given galaxy is consequently characterized by $q_{\mathrm{PAH}}, U_{\mathrm{min}}, \gamma$, and the total dust mass $M_{\text {dust }}$. In the next subsection, we describe how we derive these four parameters.

\subsubsection{Flux Ratios}

The $8 \mu \mathrm{m}, 24 \mu \mathrm{m}, 70 \mu \mathrm{m}$, and $160 \mu \mathrm{m}$ bands may be dominated by dust emission. We use the data from these four bands to determine the four parameters of the Draine \& Li (2007) model: $q_{\mathrm{PAH}}, U_{\mathrm{min}}, \gamma$, and $M_{\mathrm{dust}}$. Our approach uses the graphical procedure described in Draine \& Li (2007) to estimate the best-fit dust model. This procedure employs three flux ratios to determine the model that is the best fit to the spectral shape. The total luminosity is used to estimate the dust mass in the context of the best-fit model. The ratios defined in that paper are

$$
\begin{gathered}
P_{7.9}=\frac{\left\langle v f_{v}^{\mathrm{ns}}\right\rangle_{7.9}}{\left\langle v f_{v}\right\rangle_{71}+\left\langle v f_{v}\right\rangle_{160}} \\
P_{24}=\frac{\left\langle v f_{v}^{\mathrm{ns}}\right\rangle_{24}}{\left\langle v f_{v}\right\rangle_{71}+\left\langle v f_{v}\right\rangle_{160}} \\
R_{71}=\frac{\left\langle v f_{v}\right\rangle_{71}}{\left\langle v f_{v}\right\rangle_{160}} .
\end{gathered}
$$

The superscript "ns" refers to the nonstellar contribution to that bandpass. For the $8 \mu \mathrm{m}$ band, this is the value after subtraction of the empirical stellar contribution with the procedure described in Section 4.1. For the $24 \mu \mathrm{m}$ band, this is the value after the subtraction of the empirical stellar and circumstellar dust contribution to the $24 \mu \mathrm{m}$ band as described in Section 4.2. The measured values of these ratios are listed in Table 3 .

Not all of the galaxies with detections at $8 \mu \mathrm{m}, 70 \mu \mathrm{m}$, and $160 \mu \mathrm{m}$ have a value for $P_{7.9}$ in Table 3 because in three cases (NGC 3377, NGC 4026, and NGC 5077) the flux of the nonstellar component at $8 \mu \mathrm{m}$ is consistent with the uncertainty in the empirical scale factor and our aperture measurements, even though weak PAH emission is apparent in Figure 2. For a similar reason, no $P_{24}$ value is quoted for seven galaxies (the previous three cases, as well as NGC 2768, NGC 2787, NGC 4278, and NGC 4589) because there is more substantial variation in the empirical scale factor that corrects for the contribution from (mostly) hot, circumstellar dust in this passband.

The ratio $P_{7.9}$ is effectively a measure of the fraction of the dust emission that is radiated by PAHs, and thus provides the strongest constraint on the dust model and the $q_{\mathrm{PAH}}$ parameter. Draine \& Li (2007) note that because the $8 \mu \mathrm{m}$ emission is almost exclusively due to single-photon heating, $P_{7.9}$ is not very sensitive to the starlight intensity. The exception is that if the $\mathrm{PAH}$ fraction is very small, exposure to a more intense ISRF
Table 3

Dust Model Fits

\begin{tabular}{lccccccr}
\hline \hline Name & P7.9 & P24 & R71 & $\begin{array}{c}q \text { PAH } \\
(\%)\end{array}$ & $U_{\text {min }}$ & $\gamma$ & $\log M_{\text {dust }}$ \\
& & & & & & \\
\hline NGC 0315 & 0.13 & 0.48 & 1.47 & 2.50 & 2.0 & 0.2 & 6.7 \\
NGC 0821 & $\ldots$ & $\ldots$ & $\ldots$ & 2.50 & 7.0 & 0.0 & $<4.7$ \\
NGC 1023 & $\ldots$ & $\ldots$ & $\ldots$ & 2.50 & 7.0 & 0.0 & $<3.9$ \\
NGC 2300 & $\ldots$ & $\ldots$ & $\ldots$ & 2.50 & 7.0 & 0.0 & $<4.9$ \\
NGC 2768 & 0.06 & $\ldots$ & 2.31 & 1.12 & 15.0 & 0.0 & 5.5 \\
NGC 2787 & 0.11 & $\ldots$ & 2.52 & 1.77 & 15.0 & 0.0 & 5.2 \\
NGC 3226 & $\ldots$ & $\ldots$ & $\ldots$ & 2.50 & 7.0 & 0.0 & 5.5 \\
NGC 3377 & $\ldots$ & $\ldots$ & 0.75 & 2.50 & 2.0 & 0.0 & 4.7 \\
NGC 3414 & $\ldots$ & $\ldots$ & $\ldots$ & 2.50 & 5.0 & 0.0 & 5.6 \\
NGC 3607 & 0.18 & 0.07 & 1.53 & 3.19 & 7.0 & 0.0 & 6.3 \\
NGC 3640 & $\ldots$ & $\ldots$ & $\ldots$ & 2.50 & 7.0 & 0.0 & $<4.6$ \\
NGC 3945 & $\ldots$ & $\ldots$ & $\ldots$ & 2.50 & 7.0 & 0.0 & 5.4 \\
NGC 3998 & 0.25 & 0.44 & 1.85 & 3.90 & 7.0 & 0.15 & 5.7 \\
NGC 4026 & $\ldots$ & $\ldots$ & 1.18 & 2.50 & 5.0 & 0.0 & 5.3 \\
NGC 4138 & 0.19 & 0.13 & 1.30 & 3.19 & 5.0 & 0.02 & 6.3 \\
NGC 4278 & 0.10 & $\ldots$ & 2.09 & 1.77 & 5.0 & 0.0 & 5.4 \\
NGC 4291 & $\ldots$ & $\ldots$ & $\ldots$ & 2.50 & 7.0 & 0.0 & $<5.0$ \\
NGC 4293 & 0.10 & 0.19 & 1.65 & 1.77 & 7.0 & 0.04 & 6.5 \\
NGC 4365 & $\ldots$ & $\ldots$ & $\ldots$ & 2.50 & 7.0 & 0.0 & $<4.2$ \\
NGC 4371 & $\ldots$ & $\ldots$ & $\ldots$ & 2.50 & 7.0 & 0.0 & $<4.3$ \\
NGC 4382 & $\ldots$ & $\ldots$ & $\ldots$ & 2.50 & 7.0 & 0.0 & $<4.7$ \\
NGC 4406 & $\ldots$ & $\ldots$ & $\ldots$ & 2.50 & 7.0 & 0.0 & $<4.5$ \\
NGC 4526 & 0.12 & 0.07 & 2.35 & 2.50 & 15.0 & 0.0 & 6.4 \\
NGC 4550 & $\ldots$ & $\ldots$ & $\ldots$ & 2.50 & 7.0 & 0.0 & 4.9 \\
NGC 4570 & $\ldots$ & $\ldots$ & $\ldots$ & 2.50 & 7.0 & 0.0 & $<4.2$ \\
NGC 4578 & $\ldots$ & $\ldots$ & $\ldots$ & 2.50 & 7.0 & 0.0 & $<4.3$ \\
NGC 4589 & 0.08 & $\ldots$ & 1.37 & 1.12 & 3.0 & 0.0 & 6.1 \\
NGC 4612 & $\ldots$ & $\ldots$ & $\ldots$ & 2.50 & 7.0 & 0.0 & $<4.2$ \\
NGC 4621 & $\ldots$ & $\ldots$ & $\ldots$ & 2.50 & 7.0 & 0.0 & $<4.1$ \\
NGC 4636 & $\ldots$ & $\ldots$ & $\ldots$ & 2.50 & 7.0 & 0.0 & 4.7 \\
NGC 4649 & $\ldots$ & $\ldots$ & $\ldots$ & 2.50 & 7.0 & 0.0 & $<4.2$ \\
NGC 4694 & 0.23 & 0.14 & 1.40 & 3.90 & 5.0 & 0.02 & 6.1 \\
NGC 5077 & $\ldots$ & $\ldots$ & 1.95 & 2.50 & 12.0 & 0.0 & 5.7 \\
NGC 5273 & 0.15 & 0.28 & 2.45 & 3.19 & 15.0 & 0.06 & 5.4 \\
NGC 5308 & $\ldots$ & $\ldots$ & $\ldots$ & 2.50 & 7.0 & 0.0 & $<4.6$ \\
NGC 5557 & $\ldots$ & $\ldots$ & $\ldots$ & 2.50 & 7.0 & 0.0 & $<4.9$ \\
NGC 5576 & $\ldots$ & $\ldots$ & $\ldots$ & 2.50 & 7.0 & 0.0 & $<4.6$ \\
NGC 7619 & $\ldots$ & $\ldots$ & $\ldots$ & 2.50 & 7.0 & 0.0 & $<5.1$ \\
\hline & & & & & & &
\end{tabular}

Notes. Dust model results of the galaxies in the sample. Columns: (1) galaxy name; (2) P7.9; (3) P24; (4) R71; (5) $q_{\mathrm{PAH}}$; (6) $U_{\min }$; (7) $\gamma$; (8) $\log$ of $M_{\mathrm{dust}}$ in solar masses. Galaxies with measurements of P7.9, P24, and/or R71 were fit with the Draine \& Li (2007) models. The remaining galaxies were fit with a fixed set of parameters: $\left(q_{\mathrm{PAH}}, U_{\min }, \gamma\right)=(2.50 \%, 7.0,0.0)$. Dust mass upper limits are for this fixed model and the $3 \sigma$ upper limits at $70 \mu \mathrm{m}$ and $160 \mu \mathrm{m}$. More details are provided in Section 5.1.

(larger $\gamma$ ) will lead to enhanced PAH emission that may produce values of $P_{7.9}$ that are comparable to models with larger $q_{\mathrm{PAH}}$ and smaller values of $\gamma . P_{7.9}$ is less sensitive to $\gamma$ for larger values of $q_{\mathrm{PAH}}$ because these galaxies exhibit substantial PAH emission even when $\gamma=0$.

The value of $\gamma$ is mostly determined by the ratio $P_{24}$. This ratio is sensitive to the fraction of the dust emission near $24 \mu \mathrm{m}$ and is dominated by hot, PDR dust $(\gamma>0)$ if a hot component is present. According to Draine \& Li (2007), when larger grains are heated by starlight intensities above $U \sim 20$, they will contribute to the $24 \mu \mathrm{m}$ emission. In contrast, dust that is heated by starlight intensities of $U \sim 0.1-10$ is sufficiently cold that it emits most of its luminosity at longer wavelengths, such as the $70 \mu \mathrm{m}$ and $160 \mu \mathrm{m}$ bands. 

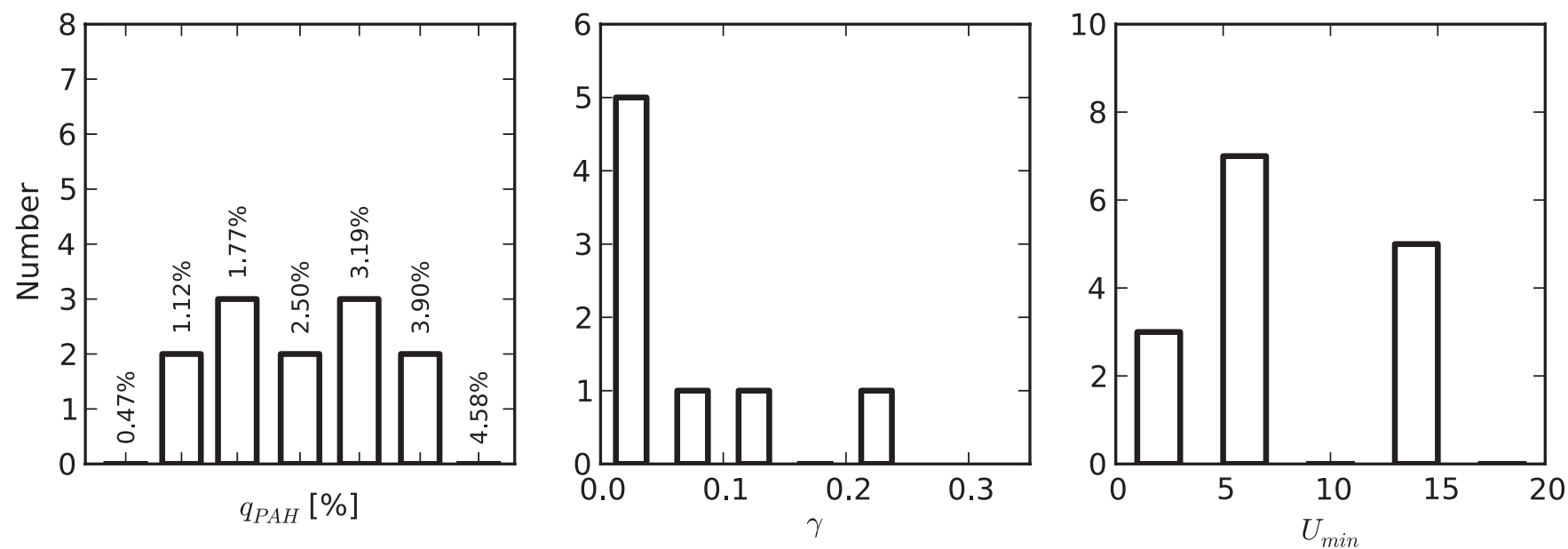

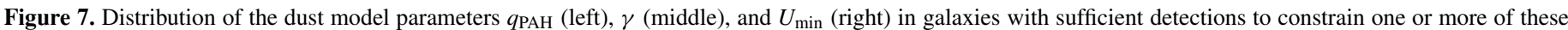
quantities. Note that NGC 3998 has $\gamma=1$ and consequently does not fall within the range shown in the middle panel.

The coldest dust component is diffusely distributed in the ISM and heated by the average ISRF of the galaxy, $U_{\min }$. The ratio $R_{71}$ is sensitive to $U_{\min }$ because the intensity of the ISRF is correlated with the characteristic temperature of the cold component, and this affects the ratio of the flux in the $70 \mu \mathrm{m}$ and $160 \mu \mathrm{m}$ bands. For small values of $\gamma$ (on order a few percent or less), the ratio $R_{71}$ is very sensitive to $U_{\min }$ because the $70 \mu \mathrm{m}$ flux has a negligible contribution from PDR dust. For larger values of $\gamma$, the PDR dust contribution to the $70 \mu \mathrm{m}$ emission is more important. In galaxies with substantial star formation, the values of both $R_{71}$ and $P_{24}$ are larger and $R_{71}$ is less sensitive to $U_{\min }$.

\subsubsection{Dust Model Parameters}

There are eight galaxies in our sample with sufficient data to constrain all three Draine \& Li (2007) model parameters. For these galaxies, we follow the graphical procedure recommended by Draine $\& \mathrm{Li}$ (2007) and first estimate $q_{\mathrm{PAH}}$ from the values of $P_{7.9}$ and $R_{71}$, and then use the values of $P_{24}$ and $R_{71}$ to estimate $\gamma$ and $U_{\mathrm{min}}$. Four additional galaxies have just $P_{7.9}$ and $R_{71}$, which is sufficient to constrain the dust model. A histogram of $q_{\mathrm{PAH}}$ for these 11 galaxies is shown in the first panel of Figure 7. The median value of $q_{\mathrm{PAH}}$ is $2.50 \%$ for these galaxies. The second panel shows the distribution of $\gamma$ for the subset of eight galaxies with sufficient coverage of the diffuse emission. The median value of $\gamma$ for these eight is 0.03 . Finally, there are three galaxies with just $R_{71}$. For these galaxies we assume $q_{\mathrm{PAH}}=2.50 \%$ and $\gamma=0$ (to provide a more conservative constraint on the dust mass than $\gamma=0.03$ ) and then estimate $U_{\min }$. The median value of $U_{\min }$ is 7.0 .

With the dust model fixed, the dust mass is

$$
M_{\text {dust }}=\frac{\Psi}{\langle U\rangle}\left(\langle v f v\rangle_{24}+\langle v f v\rangle_{71}+\langle v f v\rangle_{160}\right) D^{2}
$$

The quantity $\Psi$ is uniquely determined by the three parameters $q_{\mathrm{PAH}}, \gamma$, and $U_{\min }$ that describe the dust model, the quantity $\langle U\rangle$ accounts for starlight heating in both PDRs and the diffuse ISM, and $D$ is the distance. Dust mass histograms are shown for different morphological types in Figure 8 and for active and inactive galaxies in Figure 9.

The distributions of $q_{\mathrm{PAH}}, \gamma$, and $U_{\min }$ are generally different from the distributions found by Draine et al. (2007) for the SINGS sample. The $q_{\text {PAH }}$ values are generally lower, with the median of $q_{\mathrm{PAH}}=2.5 \%$ below the median value of $3.4 \%$ found by Draine et al. (2007) for the 61 galaxies in the SINGS sample with similar IRAC and MIPS data. The $8 \mu \mathrm{m}$ PAH feature is mostly due to ionized PAHs (Joblin et al. 1994), so the weakness of this feature may indicate a greater fraction of neutral PAHs, rather than a smaller PAH fraction. Kaneda et al. (2005) proposed this explanation based on their study of IRS spectra for four elliptical galaxies, which showed the shortwavelength PAH features were weak or absent, but the longerwavelength features at $11.3 \mu \mathrm{m}$ and $12.7 \mu \mathrm{m}$ were prominent. Their sample includes NGC 4589, for which our best model fit yields $q_{\mathrm{PAH}}=1.12 \%$, yet the longer-wavelength $\mathrm{PAH}$ features are clearly present in the IRS spectrum. Kaneda et al. (2005) suggest the relative absence of UV radiation in ellipticals could produce physical conditions amenable to primarily neutral PAHs. Such conditions are also indicated by the SED of the bulge of M31 by Groves et al. (2012).

Other potential explanations include the preferential destruction of small particles, such as due to sputtering in hot gas or an AGN, and low gas-phase metallicities. The dominance of larger PAHs over smaller ones, which could be a consequence of sputtering (Schutte et al. 1993; Tsai \& Mathews 1995; Micelotta et al. 2010), would weaken the $8 \mu \mathrm{m}$ feature. The $q_{\mathrm{PAH}}$ value is also observed to decline in galaxies with low gas-phase oxygen abundance. Draine et al. (2007) find that the median PAH fraction is $1.0 \%$ for galaxies with gas-phase oxygen abundances below $12+\log _{10} \mathrm{O} / \mathrm{H}<8.1$ (it is also low for a small fraction of higher metallicity galaxies). Even though our sample is expected to possess solar to supersolar stellar abundances, the cold gas abundance may be quite low if it is the remnant of an accreted, low-mass satellite. Finally, relatively luminous AGNs have also been implicated in the destruction of PAHs (Lutz et al. 2008) and all but three of the galaxies with detections in all seven bands are AGNs, although they are low-luminosity AGNs. Smith et al. (2007) found that AGNs may modify the relative distribution of intensities of various PAH features, and in particular suppress those from 5-8 $\mu \mathrm{m}$. However, low-luminosity AGNs do not appear to negatively affect the PAH abundance, as Draine et al. (2007) found that the median $q_{\text {PAH }}$ fraction was actually slightly higher $(3.8 \%)$ for AGNs than the median of their full sample, as well the median of 28 galaxies with $\mathrm{H}$ II nuclei $(3.1 \%)$.

Nearly all of the galaxies have $\gamma<0.1$ and the median value is $\gamma=0.03$. This is consistent with the near-absence of star formation in these galaxies. The small values of $\gamma$ also mean that the model fits and dust masses are more reliable, as in this 


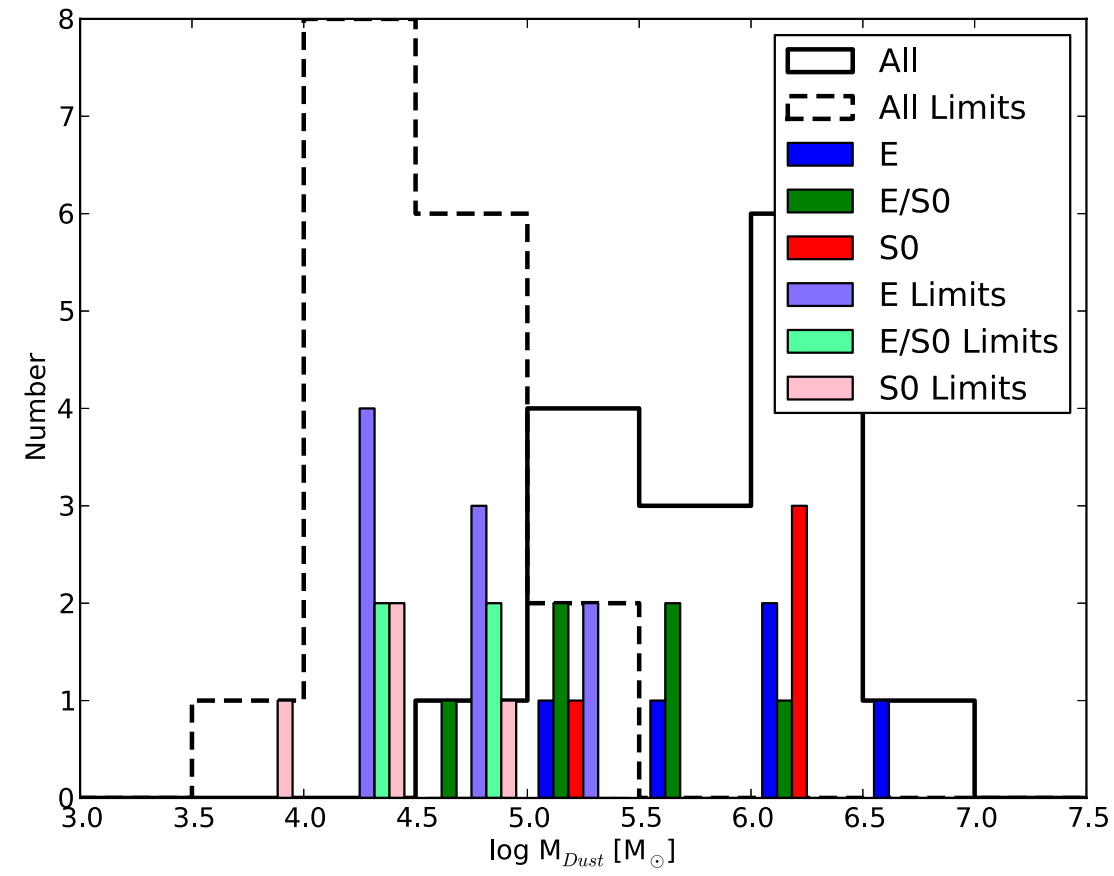

Figure 8. Distribution of dust masses and upper limits as a function of morphological type.

(A color version of this figure is available in the online journal.)

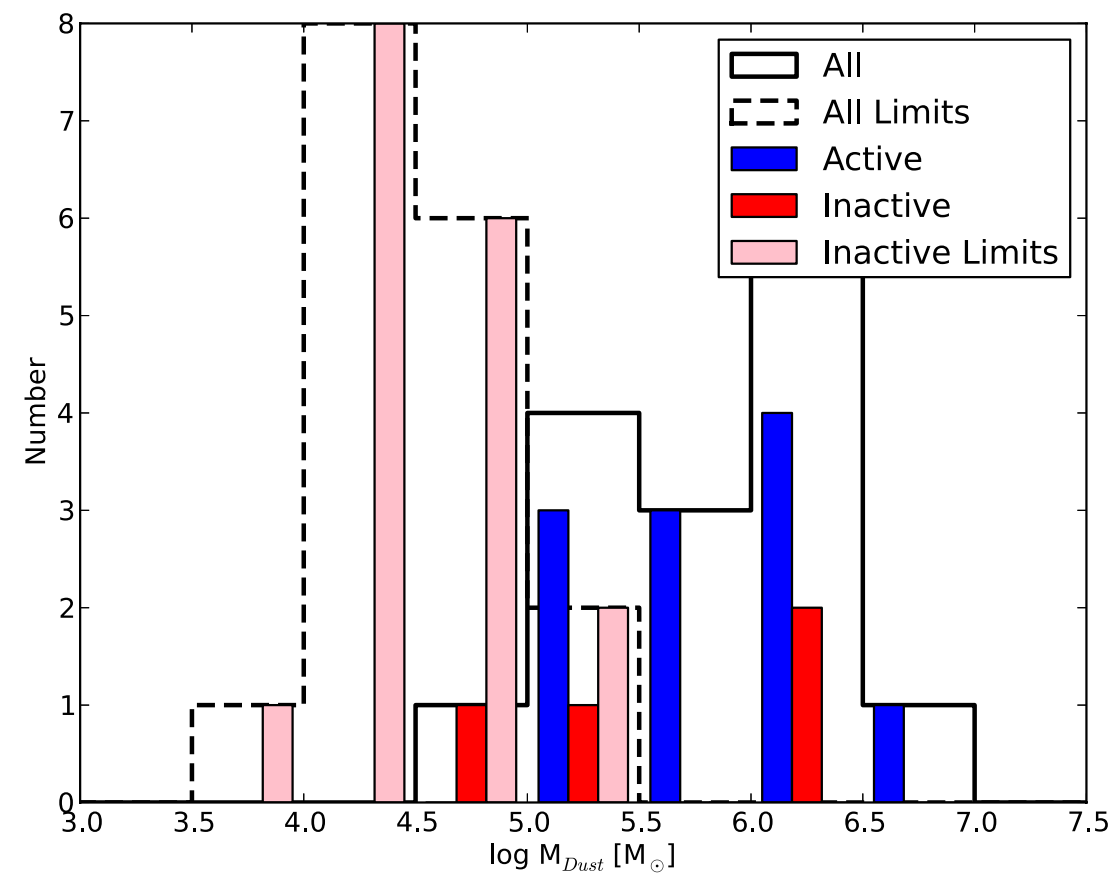

Figure 9. Distribution of dust masses and upper limits as a function of nuclear activity.

(A color version of this figure is available in the online journal.)

regime the parameter $R_{71}$ provides a better estimate of $U_{\text {min }}$. The median $U_{\min }$ value for this sample is 7.0, which is several times higher than the SINGS sample median of 1.5. The median for the subsample of SINGS galaxies with SCUBA data is in better agreement with our data. In the case of SINGS galaxies with SCUBA observations, the cold and PDR dust components are better separated, and thus $U_{\min }$ is better measured for those galaxies. We may be able to measure larger values of $U_{\text {min }}$ without longer-wavelength submillimeter observations because our galaxies have lower values of $\gamma$. Early-type galaxies may also have more intense ISRFs than spiral galaxies due to the presence of hot, evolved stars and higher stellar densities. This is also suggested by their higher dust temperatures relative to spirals (Bendo et al. 2003; Skibba et al. 2011; Smith et al. 2012; Auld et al. 2013). Emission from hot, evolved stars contributes to and may dominate the LINER emission spectra from some galaxies (Sarzi et al. 2010). Longer-wavelength observations would help further constrain the value of $U_{\min }$, while far-infrared line ratios would help to constrain the spectral shape of the ionizing photons (e.g., Malhotra et al. 2000).

An important caveat that affects the interpretation of the dust model parameters is that uncertainties, or intrinsic variations, in 
the empirical scale factors derived in Section 4.1 increase the uncertainties in the dust model parameters. As noted previously, the data shown in Figures 3 and 5 exhibit evidence for intrinsic variation in these quantities, and some variation is expected due to stellar population differences. This is particularly relevant for $P_{7.9}$ and $P_{24}$, which largely determine $q_{\mathrm{PAH}}$ and $\gamma$, and so these parameters are not as well constrained as $U_{\min }$. In practice, as $\gamma$ is expected to be small due to the general absence of star formation in these galaxies, the biggest potential uncertainty lies with $q_{\text {PAH }}$.

The measured dust masses for our sample are $M_{\text {dust }} \sim$ $10^{5}-10^{6.5} M_{\odot}$, which are two or three orders of magnitude lower than the dust masses for spiral galaxies of comparable size, but similar to other early-type galaxies (Goudfrooij \& de Jong 1995; Draine et al. 2007; Smith et al. 2012). The dust masses that result from this procedure are fairly robust, even though they are only based on measurements out to $160 \mu \mathrm{m}$. This is true even if the model parameters $q_{\mathrm{PAH}}, \gamma$, and $U_{\min }$ are not well constrained by the data because $q_{\mathrm{PAH}}$ has a negligible impact on the dust mass estimate and $\gamma=0$ is a good approximation for most galaxies. For the full range of models presented in Draine \& Li (2007), we also note that the value of $\Psi$ that is set by the best-fit model only varies from

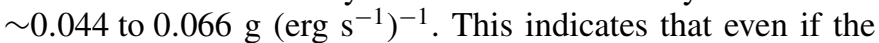
dust model were not constrained at all by the data and one simply adopted the average of this range, there would only be a $\sim 20 \%$ uncertainty in the dust mass within the context of these models. An additional uncertainty is the ISRF because the absence of flux measurements at wavelengths longer than $160 \mu \mathrm{m}$ do not constrain a potentially substantial mass of cold dust. However, the available data can constrain the ISRF quite well because these galaxies have little star formation. Another estimate of the uncertainties comes from the overlap between several galaxies in our study and previous investigations with Infrared Space Observatory (Xilouris et al. 2004), Spitzer (Temi et al. 2007), and longer-wavelength Herschel data (Smith et al. 2012; Auld et al. 2013). The dust masses derived by those previous studies assumed a modified blackbody and mostly agree with our estimates to within a factor of two. The noteworthy exception is NGC 3945, for which Smith et al. (2012) derive over an order of magnitude larger dust mass. The difference could be ascribed to the fact that we use a smaller aperture, although our $70 \mu \mathrm{m}$ flux is within $5 \%$ of the IRAS $60 \mu \mathrm{m}$ value. However, due to the large uncertainty in this IRAS measurement it is still possible that we are missing extended emission from dust in this object. We may be able to put tighter constraints on the dust mass for this object with future analysis of Herschel photometry. We also note we were unable to obtain a good measurement of NGC 3945 at $160 \mu \mathrm{m}$. We do retain NGC 3945 in our sample. Another comparison was performed by Gordon et al. (2010), who found that dust masses estimated from $\leqslant 160 \mu \mathrm{m}$ data can differ by $10 \%-36 \%$ relative to dust mass estimates that include Herschel measurements at $250 \mu \mathrm{m}$ and $350 \mu \mathrm{m}$, although this study was based only on observations of the Large Magellanic Cloud and therefore does not include uncertainties due to differences in grain size and ISRF intensity distributions. Nevertheless, this study and a recent one by Aniano et al. (2012) of two nearby spirals that also compared Spitzer and Herschel provide good evidence against substantial masses of cold dust.

A final uncertainty is that the physical dust model that works so well for the Milky Way may not be appropriate for early-type galaxies, that is, the size and composition are different because of different formation and processing histories. For example, sputtering can reduce the fraction of small grains relative to the initial grain size distribution. The generally smaller values of $q_{\mathrm{PAH}}$ in early-type galaxies relative to spirals may indicate that this is the case, although there is also allowance for this difference in the Draine \& Li (2007) models. The study of four ellipticals by Kaneda et al. (2005) also provides some evidence that the shape of the radiation field is softer in ellipticals than in the Milky Way, which may not be adequately compensated by an adjustment in $U_{\min }$. Provided all early-type galaxies are different in the same manner, then the relative dust masses we have derived here should nevertheless be robust. The true uncertainties in the dust masses are likely dominated by these model details and are difficult to quantify. Under the assumption that the dust model is a reasonably good match to early-type galaxies, we estimate that the uncertainties in the dust masses are on order a factor of two or three due to a combination of aperture differences, calibration uncertainties, and differences (or uncertainties) in the dust models.

\subsubsection{Upper Limits}

Approximately half of the galaxies in our sample are not detected at $70 \mu \mathrm{m}$ and $160 \mu \mathrm{m}$. These non-detections nevertheless provide very useful constraints on the total dust mass in these galaxies because of the extraordinary sensitivity of the Spitzer MIPS instrument. To calculate upper limits on the dust masses in these galaxies, we employ a dust model with $q_{\mathrm{PAH}}=2.50 \%$, $\gamma=0$, and $U_{\min }=7.0$. The values of $q_{\mathrm{PAH}}$ and $U_{\mathrm{min}}$ are representative of the galaxies with the best data (see Figure 7). We chose to use $\gamma=0$, rather than the median value of $\gamma=0.03$, because this leads to a more conservative constraint on the dust mass.

We use this dust model to calculate the largest value of the dust mass that is consistent with the $3 \sigma$ upper limits at $70 \mu \mathrm{m}$ and $160 \mu \mathrm{m}$. These upper limits are listed in Table 3 and shown in Figures 8 and 9. The figures clearly show that the upper limits on the dust masses are approximately an order of magnitude lower than the measured dust masses.

All of these upper limits are for galaxies that show no evidence of dust lanes within hundreds of parsecs of their nuclei based on our earlier HST study (Simões Lopes et al. 2007, and also shown in the leftmost panels of Figure 2). While the structure map technique is insensitive to uniformly distributed or very diffuse dust, the Spitzer data place very strong upper limits on the mass of such dust. The PAH images are also valuable in this respect, as we describe in the next subsection.

\subsection{PAHs}

As a second search for diffuse, extended dust that may not have been present in our HST study, we created PAH images for all of the galaxies. These images are shown in the middle panels of Figure 2 . The PAH images are the $8 \mu \mathrm{m}$ image minus a scaled version of the $4.5 \mu \mathrm{m}$ image, where the scale factor accounts for the relative stellar emission in the two images. The calculation of the scale factor is described in Section 4.1. The pair of images were convolved to the same PSF prior to subtraction. All of the galaxies with dust lanes in the HST structure maps also have clear PAH emission, with the exception of NGC 4636, which has only marginally detected dust lanes in the HST data. Conversely, none of the galaxies with no evidence for dust at visible wavelengths exhibit clear evidence of PAH emission. A number of the PAH images of the galaxies exhibit artifacts at their centers. These are consistent with artifacts of the stellar 
continuum subtraction, which could be caused by uncertainties in the scale factor, the PSF shape, and centroid errors.

There is a good correspondence between the morphology of the dust lanes in the HST data and the distribution of PAH emission for the small subset of galaxies where the HST data are of sufficient quality and the dust lanes sufficiently extended. Some particularly striking examples are NGC 4138 and NGC 4293. In a few cases, the structure maps show substantial asymmetries in the dust distribution that are not mirrored in the PAH images. The most likely explanation is that the dust lanes viewed in absorption are substantially less prominent on the side of the galaxy that is tilted away from our perspective (e.g., Hubble 1943).

\subsection{Distribution of Dust Masses}

The dust mass distribution shown in Figure 8 shows that most detections have $10^{5}-10^{6} M_{\odot}$ of dust. There are only three detections below $10^{5} M_{\odot}$ (NGC 3377, NGC 4550, and NGC 4626), while there are many upper limits that extend an order of magnitude lower and suggest a relative absence of galaxies with $<10^{5} M_{\odot}$ of dust. For example, if there were as many galaxies with dust masses in the range $10^{4}-10^{5} M_{\odot}$ as in the range $10^{5}-10^{6}$, we would expect ten galaxies in this lower range and that we would detect half of them, yet only one is detected. This apparent dichotomy is also supported by the similar dichotomy in the detection of dust lanes in HST images of these galaxies (see Table 1), as we do not expect the Spitzer and HST images to have comparable dust mass sensitivity.

One possible explanation is a difference in morphological type. Figure 8 splits the detections and upper limits by morphology into ellipticals, S0s, and E/S0s. We see no evidence that true ellipticals or true S0 galaxies are more or less likely to have interstellar dust. The sensitivity of the data sets for each morphological type are also comparable. The upper limits on the dust mass in undetected ellipticals and lenticulars both span the full range of upper limits from approximately $10^{4}-10^{5} M_{\odot}$. We used the Astronomical Survival Analysis (ASURV) programs from Feigelson \& Nelson (1985), as implemented in the IRAF STSDAS STATISTICS package, to compare the elliptical and lenticular subsamples. We ran five different tests through the twosampt task and these indicated that the distributions are consistent. These similar distributions are in contrast to the results of Smith et al. (2012) with the Herschel Reference Survey, who detected dust in $24 \%$ of ellipticals and $62 \%$ of S0s with SPIRE. This difference in detection rate between the two morphological types could be due to their larger sample of 62 early-type galaxies. Another potential explanation is that Cortese et al. (2012) found that the dust to stellar mass ratio is lower for ellipticals than lenticulars. If our ellipticals were more massive than our lenticulars, this would also explain their similar dust masses.

We investigated if there is a correlation between dust detections or upper limits and cluster membership. Environment may correlate with the absence of dust because the hot atmospheres of clusters may lead to an increase in the rate of dust destruction, although many cluster early-type galaxies do maintain their own atmospheres (e.g., Sun et al. 2005). With regard to environment, clusters of galaxies have substantially fewer gas-rich dwarf galaxies and sufficiently high relative velocities that mergers are more rare. We searched for differences as a function of environment, and whether or not environment could explain the dust dichotomy, with the 14 Virgo members and 27 nonmembers in our sample and detect dust in 18/27 (66.6\%) nonmembers, compared to $5 / 14(45.4 \%)$ Virgo members. This difference suggests a slightly larger incidence of dust in galaxies outside of Virgo, although it is not statistically significant. Smith et al. (2012) performed a similar analysis for the earlytype galaxies in the Herschel Reference Survey and found a similar trend, but that it was also not statistically significant. We also investigated whether or not membership influenced the detection of dust in the inactive sample. We detect dust in 2 of the 11 inactive Virgo members, compared to three out of 11 of the inactive galaxies outside of Virgo. There is thus no evidence that membership in the Virgo Cluster is correlated with a lower incidence of dust in inactive galaxies.

We also compared the dust detection rate with stellar luminosity, which correlates well with stellar mass. More massive galaxies generally have higher ISM temperatures, which will lead to faster dust destruction rates. This naively suggests an anticorrelation between dust mass and stellar luminosity, although no such anticorrelation is present in our data. In Figure 10, we show the dust masses and upper limits as a function of $L_{4.5}$, a proxy for stellar mass. While the minimum detected dust mass is a function of stellar luminosity, this is because more luminous galaxies are more rare and thus at larger distances.

The dichotomy between the dust mass estimates and upper limits thus suggests that there are distinct populations of dusty and non-dusty early-type galaxies, rather than a continuum of dust masses. To explore this possibility further, we resampled our data many times in order to determine if the apparent dichotomy is due to the sensitivity distribution of our data, rather than an intrinsically nonuniform distribution of dust masses. To perform these calculations, we estimated the minimum flux sensitivity of the $160 \mu \mathrm{m}$ data for each galaxy. We then generated a uniform distribution in $\log M_{\text {dust }}$, randomly assigned dust masses from this distribution to galaxies in our sample, calculated the flux at $160 \mu \mathrm{m}$ based on a randomly selected galaxy's distance for the dust model used to estimate upper limits, and determined if this flux would correspond to a detection or upper limit based on the sensitivity of the same galaxy's data set. We generated 1000 realizations of our data set and found that the sensitivity and distance distribution of our data set does not produce the observed dichotomy between detections and upper limits for an intrinsically uniform distribution in $\log M_{\text {dust }}$ approximately $\sim 95 \%$ of the time.

While this is formally marginally significant, an important caveat to this statistical analysis is that it does not include variations due to the best-fit dust model, which may add a factor of two uncertainty to the dust mass, with the possibility that we may systematically underestimate the dust mass by tens of percent in some cases, particularly for nearby galaxies with extended emission, and potential correlations with morphology, stellar mass, and environment. We plan to re-examine this potential dichotomy with a larger sample.

\subsection{Correlation with AGNs}

The distribution of dust masses is strikingly different for active and inactive galaxies. Galaxies classified as active, that is, either LINERs or Seyferts according to Ho et al. (1997a), are all detected and have dust masses that range from $M=10^{5}-10^{6.5} M_{\odot}$. In contrast, only four galaxies classified as inactive are detected and the remaining 16 have upper limits that range from $M \sim 10^{4}-10^{5} M_{\odot}$. This result is similar to the strong correlation between activity and the presence of dust structure within the central kpc in our earlier HST study (Simões Lopes et al. 2007), yet these new observations demonstrate that the galaxies without dust lanes have at least one to two orders 


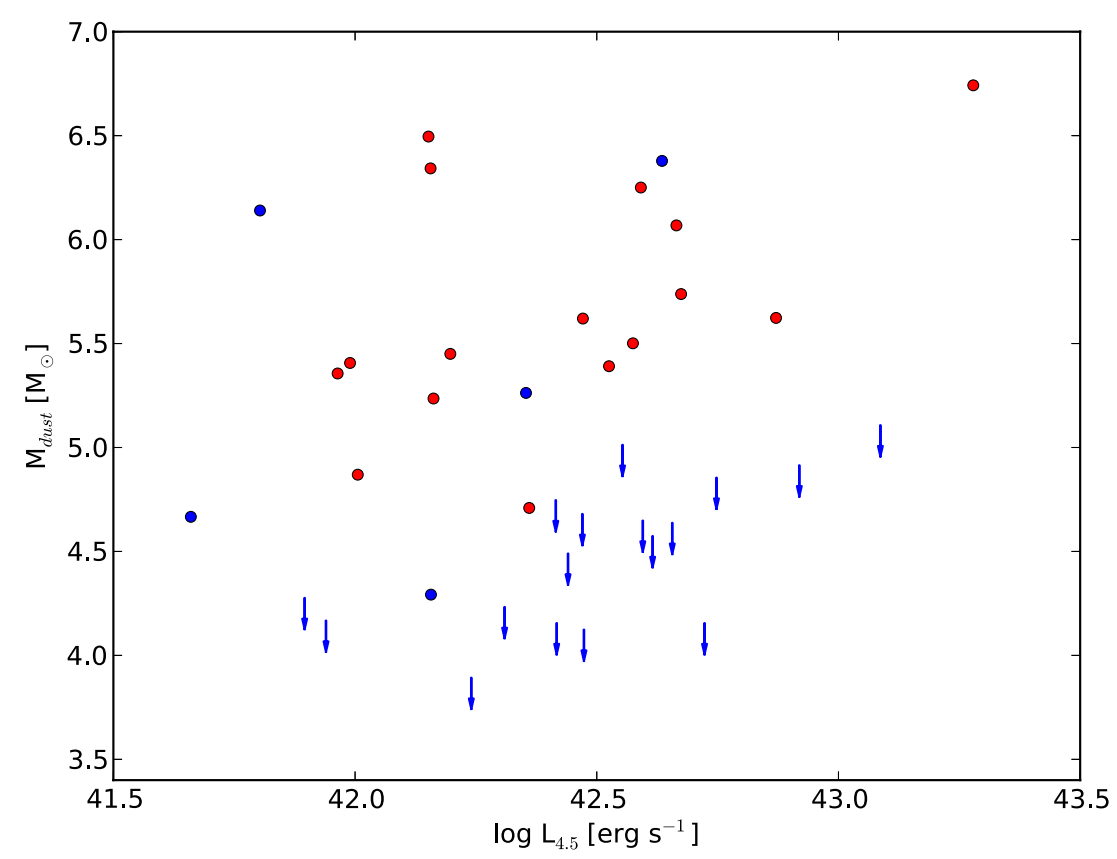

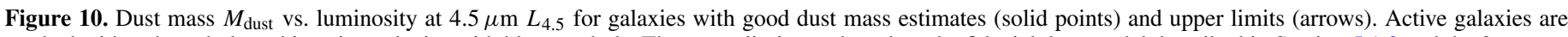

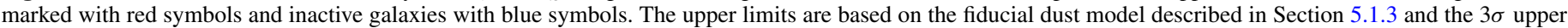
limits on the flux at $70 \mu \mathrm{m}$ and $160 \mu \mathrm{m}$.

(A color version of this figure is available in the online journal.)

of magnitude less dust than those with detections. As in the previous section, we used the ASURV programs from Feigelson $\&$ Nelson (1985) to compare the dust mass distributions of the active and inactive samples. All five tests available through the twosampt task returned zero probability that the dust masses were drawn from the same distribution.

There has been substantial discussion in the literature of the extent to which the emission lines characteristic of LINERs are actually powered by accretion onto supermassive black holes. Other proposed candidates are fast shocks, interaction with the hot phase of the ISM, and photoionization by UV emission from old stellar populations (see Ho 2008, for a review). Sarzi et al. (2010) performed a detailed analysis of emission-line ratios for many early-type galaxies and concluded that the emission-line luminosities of only a minority of LINERs are dominated by accretion. Nevertheless, AGNs have been confirmed in the majority of LINERs based on measurements of radio cores, X-ray cores, and the surface brightness profiles of various emission lines relative to the stellar continuum (Filho et al. 2004, 2006; Nagar et al. 2005; Flohic et al. 2006; Ho 2008; Sarzi et al. 2010). Some fraction of the cold ISM in the majority of these dusty early-type galaxies is therefore fueling their central, supermassive black holes, even if their emissionline luminosities are not dominated by nuclear accretion. We hypothesize that the correspondence between active galaxies and dust in our sample is simply due to the presence of a sufficient quantity of cold material, some of which has accreted onto the black hole, and some of which is ionized and produces the visible-wavelength emission lines, rather than a causal relationship between the presence of dust and AGNs.

\section{ORIGIN OF THE DUST}

The main motivation for this study was to use dust mass estimates for early-type galaxies to constrain the origin of their dust. Our estimates demonstrate that dusty early-type galaxies have $10^{5}-10^{6.5} M_{\odot}$ of dust, while the remainder have at least one order of magnitude less. Our sample is representative of the early-type galaxy population as a whole because the original selection of the sample was from the magnitude-limited Palomar Survey of Ho et al. (1995). In Simões Lopes et al. (2007), we selected an equal number of active and inactive galaxies from all of the early-type galaxies in the Palomar survey that had visible-wavelength $H S T$ images. Half of this sample were active galaxies and half were not, which is equivalent to the relative frequency of active and inactive galaxies in the early-type galaxy population. Simões Lopes et al. (2007) then found that all of the galaxies classified as active had dust lanes within the central kpc, whereas only $25 \%$ of the inactive galaxies had them. While we have more inactive galaxies than active galaxies in this sample, we still find that all active galaxies have interstellar dust and find a similarly small fraction $(5 / 22$ or $23 \%)$ of the inactive galaxies have dust. We therefore conclude that $\sim 60 \%$ of all early-type galaxies have dust, including all of those classified as active, and $\sim 40 \%$ do not.

The presence of dust in any early-type galaxy has long been a puzzle because thermal sputtering of dust grains in the hot $\left(T>10^{6} \mathrm{~K}\right)$ gas that comprises the bulk of their ISM should destroy dust on the timescale $\tau_{\text {dust }} \sim 2 \times 10^{4}$ yr (Draine \& Salpeter 1979b). These galaxies clearly do have evolved stars that produce dust, as indicated by the strong evidence for hot, circumstellar dust, and this dust must be ejected into the ISM at an approximately constant rate due to the age of their stellar populations. The expected rate of mass loss, the dust-to-gas ratio of this material, and the dust destruction timescale, can be used to estimate the steady-state dust mass of these galaxies. As noted in the Introduction, for reasonable estimates of the mass-loss rate, dust-to-gas ratio, and dust destruction time in hot gas, the steady-state dust mass should be $10-100\left(\tau_{\text {dust }} / 2 \times 10^{4} \mathrm{yr}\right) M_{\odot}$, that is, none of the galaxies should have been detected.

The dust destruction timescale could be longer if the temperature of the hot phase is closer to $10^{5} \mathrm{~K}$, or if some of 
the gas and dust cools rapidly and settles into a cooler phase (Mathews \& Brighenti 2003), although the dust destruction timescale needs to be many orders of magnitude longer to produce dust masses of $10^{5} M_{\odot}$ and above. A further complication is that this calculation predicts the expected steady-state dust mass for all early-type galaxies. As early-type galaxies form a fairly homogeneous population, all early-type galaxies should have similar dust production rates and destruction timescales. The internal origin model is consequently inconsistent with the order-of-magnitude differences in dust mass for similar galaxies, such as is illustrated by Figure 10.

The many galaxies with upper limits on the dust mass provide an upper limit on the typical dust destruction timescale in the hot ISM through an inversion of the argument presented above. For typical early-type galaxies, the most stringent upper limits on the dust mass are approximately $M_{\text {dust }}<10^{4}-10^{4.5}$. For a continual dust production rate of $(0.1-1) \times 0.005=$ $(5-50) \times 10^{-4} M_{\odot} \mathrm{yr}^{-1}$, the upper limit on the dust destruction timescale is

$$
\tau_{\text {dust }}<2 \times 10^{7}\left(\frac{M_{\text {dust, lim }}}{10^{4}}\right)\left(\frac{0.1}{\dot{M}_{\text {gas }}}\right)\left(\frac{0.005}{\epsilon_{\text {dg }}}\right) \mathrm{yr},
$$

where $M_{\text {dust,lim }}$ is the upper limit on the dust mass, $\dot{M}_{\text {gas }}$ is the gas mass-loss rate of the evolved stellar population, and $\epsilon_{\mathrm{dg}}$ is the dust-to-gas ratio. This upper limit is comparable to the value of $\tau_{\text {dust }}<46 \pm 25$ Myr derived by Clemens et al. (2010) with a similar argument, as well as more recent work by Smith et al. (2012). Both values are consistent with, although several orders of magnitude greater than, the calculations of Draine \& Salpeter (1979b), and inconsistent with an internal origin for the one to two orders of magnitude more dust present in approximately half of the sample. As noted in the Introduction, a number of previous studies have also pointed out the difficulties with a purely internal origin (Simões Lopes et al. 2007; Rowlands et al. 2012). In particular, the recent study by Smith et al. (2012) reached a similar conclusion with a similar analysis of 62 earlytype galaxies observed by Herschel.

The alternative, and favored, hypothesis for the origin of the dust is the accretion of gas-rich satellites (e.g., Tran et al. 2001; Verdoes Kleijn \& de Zeeuw 2005). The morphology of the dust lanes observed in HST images indicates that many of them are chaotic and clumpy and this appears inconsistent with steady accumulation from stellar mass loss (van Dokkum \& Franx 1995; Simões Lopes et al. 2007). Furthermore, when smooth dust disks are present, they do not always share the major axis of the host galaxy. Kinematic observations of atomic (e.g., Bertola et al. 1984, 1992; Sarzi et al. 2006; Morganti et al. 2006) and molecular (Combes et al. 2007; Davis et al. 2011) gas in earlytype galaxies also indicate that the gas kinematics are often not aligned with the kinematics of the stars, which further suggests an external origin.

The key challenges to the external origin hypothesis are that the merger rate of gas-rich satellites is low and the dust destruction timescale is short, yet dust is present in slightly more than half of all early-type galaxies. That is, the external origin model must satisfy the constraint

$$
f_{\text {dust }}=\Re_{\text {merg }} \tau_{\text {dust }},
$$

where $f_{\text {dust }}=0.6$ is the fraction of the population with dust above some minimum mass, $\mathfrak{R}_{\text {merg }}$ is the merger rate of gasrich satellites that could supply sufficient dust, and $\tau_{\text {dust }}$ is the dust destruction time. Dwarf galaxies with $10^{5}-10^{6} M_{\odot}$ of dust (comparable in size to the Magellanic clouds) would correspond to 1:300-1:100 mass-ratio mergers for the galaxies in this sample. Based on the equations provided by Stewart et al. (2009), we calculate that the cumulative merger rate for mass ratios from equal-mass mergers to 1:300 mergers is $\Re_{\text {merg }}=0.07-0.2 \mathrm{Gyr}^{-1}$, which is more than two orders of magnitude higher than our upper limit of $\tau_{\text {dust }}<0.02$ Gyr and more than four orders of magnitude greater than the theoretical estimate (Draine \& Salpeter 1979b). These values predict $f_{\text {dust }}<0.0014-0.004$, or a discrepancy of 150-430.

To evaluate the significance of this discrepancy, we estimate the uncertainty in both quantities. First, the merger rate we have adopted may be an overestimate because the theoretical calculations predict the merger rates for all galaxies as a function of stellar mass, yet not all satellite galaxies may have sufficient cold gas. It is also possible that the theoretical estimates underestimate the true merger rates. Observational evidence for an underestimate was presented by Lotz et al. (2011), who found that the observed merger rates are approximately a factor of five higher than the predictions of Stewart et al. (2009) and other recent, theoretical analyses. Lotz et al. (2011) posit that the disagreement between theory and observation could be because the visibility timescale for the mergers has been overestimated, or the theoretical rate has been underestimated. While the merger visibility timescale is arguably more uncertain that the merger rates from simulations, this comparison indicates the merger rates are unlikely more than a factor of a few larger than the theoretical estimates. There is consequently insufficient uncertainty in the merger rates to resolve this discrepancy.

The other potential resolution is that the dust destruction timescale is actually substantially higher than both the theoretical estimate of Draine \& Salpeter (1979b) and our empirical upper limit. While this requires an increase of about two orders of magnitude relative to the upper limit, the dust destruction timescale is much more uncertain than the merger rate. One reference value is the dust destruction timescale for the Milky Way $\tau_{\text {dust,Mw }}=0.4 \mathrm{Gyr}$, which is dominated by the impact of supernova shocks on the cold, neutral medium (Barlow \& Silk 1977; Draine \& Salpeter 1979a; Dwek \& Scalo 1980; Jones et al. 1994). As the supernova rate in early-type galaxies is several times lower than in the Milky Way (e.g., Li et al. 2011), the dust destruction timescale may be several times larger in their cold ISM and plausibly on the order of a Gyr, provided the accreted dust does not mix with the hot ISM. This may be the case if a much larger dust destruction timescale, combined with the highest estimated merger rates, predicts a dusty fraction that is only a factor of a few below what is needed to reproduce the observations. This is plausibly within the uncertainties of these order-of-magnitude estimates, particularly if dust destruction is very inefficient in the cold ISM of early-type galaxies and the cold ISM does not mix efficiently with the hot phase. However, it is also plausible that the lifetime will be less because there is less cold ISM. A more precise estimate could be obtained with an analysis of the fraction of the supernova energy that impacts the cold ISM.

While it may be possible for purely external accretion to work, we instead propose that the solution is a hybrid of internal production and external accretion. When early-type galaxies accrete a gas-rich satellite, they accrete substantial amounts of both cold gas and dust. While dust will be destroyed by a combination of supernova shocks and sputtering, this destruction could be balanced by continued dust grain growth 
in the accreted cold ISM. While this process is not constrained by observations of early-type galaxies, many studies of the Milky Way have concluded that $>90 \%$ of dust formation by mass occurs in the cold ISM (Draine \& Salpeter 1979a; Dwek \& Scalo 1980; McKee 1989; Draine 2009), rather than from stellar sources such as mass loss and supernova alone (Barlow et al. 2010; Matsuura et al. 2011; Dunne et al. 2011). The Milky Way has $M_{\text {gas }}=5 \times 10^{9} M_{\odot}$ of cold gas that produces $M_{\text {dust }}=2.5 \times 10^{7} M_{\odot}$ per dust destruction timescale, which corresponds to a steady-state dust mass of $\epsilon_{\mathrm{dg}} M_{\mathrm{gas}}$. While the dust destruction timescale is likely different from the Milky Way value, the steady-state dust mass is likely to be within an order of magnitude of that predicted by the product of the Milky Way dust-to-gas ratio and the observed cold gas supply.

Continued dust production in the accreted cold gas removes the main weakness of the internal origin hypothesis because the mass of neutral gas in early-type galaxies varies by orders of magnitude (e.g., Welch et al. 2010), even though their stellar populations are quite similar. The hybrid solution also removes the main weakness of the external origin hypothesis because dust destruction is at least partially balanced by growth in the cold gas. As a result, the timescale that is important to explain the demographics of dust is the cold gas depletion timescale, rather than the destruction timescale of individual grains. As the depletion timescale for the cold gas appears to be on the order of Gyr, largely due to star formation, it is sufficient to maintain substantial quantities of dust in approximately half of all early-type galaxies.

This hybrid solution leads to several testable predictions. First, the cold gas in early-type galaxies should be lower metallicity than the old stellar population, which may be accessible with gas-phase abundance measurements. Second, the dusty early-type galaxies should have cold gas to dust ratios comparable to the accreted satellite population, unless the cold gas and dust are destroyed at different rates. Finally, there should be more evidence of mergers within the last few Gyr in the dusty early-type galaxies than those that appear dust free.

Both external accretion and our hybrid solution also constrain the lifetime of the low-luminosity AGN in the dusty early-type galaxies. If the only source of the cold gas and dust that fuels the supermassive black hole is external accretion, and these accretion events only occur every few Gyr, the high incidence of AGNs implies that these galaxies remain (weakly) active for several Gyr as well, while the inactive galaxies remain inactive for comparable timescales. The mass of dust, and the two orders of magnitude more cold gas in the ISM, is more than sufficient to maintain accretion rates of $\leqslant 0.01 M_{\odot} \mathrm{yr}^{-1}$ for this time, as well as fuel the modest circumnuclear star formation necessary to produce the nuclear stellar disks seen in many dust-free earlytype galaxies (e.g., Simões Lopes et al. 2007).

\section{SUMMARY}

We have analyzed the dust in a representative sample of 38 early-type galaxies and used these data to constrain the origin of their dust. This analysis is largely based on archival Spitzer IRAC and MIPS observations, although these galaxies also have $H S T$ observations that reveal the presence or absence of dust lanes in the central kpc, as well as high $\mathrm{S} / \mathrm{N}$, visible-wavelength spectroscopy that provides uniform classifications of nuclear activity.

The IRAC and MIPS observations detect emission from dust from every galaxy that exhibits dust lanes seen in absorption in
HST observations. This dust emission is clearly due to both PAH particles, as detected with IRAC $8 \mu \mathrm{m}$ observations, and cold interstellar dust, as detected by MIPS observations at $70 \mu \mathrm{m}$ and $160 \mu \mathrm{m}$. Conversely, galaxies that show no evidence for dust lanes in HST observations also do not exhibit dust emission at longer wavelengths. The absence of dust emission indicates that galaxies without clumpy dust lanes within hundreds of parsecs of their centers do not have a substantial quantity of uniform, diffusely distributed dust that would have been difficult to detect with contrast enhancement techniques.

The galaxies with no evidence for interstellar dust are a valuable sample to estimate the stellar SED for old stellar populations. We use these data to estimate the ratio of the stellar emission at $8 \mu \mathrm{m}$ to that at $3.6 \mu \mathrm{m}$ and $4.5 \mu \mathrm{m}$. We then use these flux ratios to scale and subtract the stellar contribution from the $8 \mu \mathrm{m}$ band for the galaxies with interstellar dust and obtain a good estimate of the emission from PAHs in this bandpass. These flux ratios are in reasonable agreement with spectral synthesis models of old stellar populations.

We also used the galaxies without interstellar dust to estimate the ratio of the flux in the $24 \mu \mathrm{m}$ MIPS band to the shorterwavelength, photospheric emission at $3.6 \mu \mathrm{m}$ and $4.5 \mu \mathrm{m}$. The ratio of the $24 \mu \mathrm{m}$ emission to these two shorter-wavelength bands is fairly constant across galaxies of a wide range of luminosity, yet the ratio exceeds that expected from photospheric emission by approximately a constant factor of four. We conclude that the discrepancy is because emission from hot dust in the circumstellar envelopes of evolved stars makes a substantial contribution to the $24 \mu \mathrm{m}$ band. We use this ratio to remove the circumstellar dust contribution from the early-type galaxies that have dust in order to study the dust properties of the ISM.

A number of these galaxies have sufficient diffuse dust emission that we can determine one or more of the dust model parameters introduced by Draine \& Li (2007). These are the PAH fraction $q_{\mathrm{PAH}}$, the fraction of the dust heated by a strong radiation field $\gamma$, and the minimum starlight intensity $U_{\min }$. The best-fit model and the far-infrared luminosity then determine the dust mass. This analysis indicates that early-type galaxies are usually well fit by the same dust models that work well for spiral galaxies. We do find evidence that early-type galaxies have slightly lower values of $q_{\mathrm{PAH}}$, which may be due to the presence of primarily neutral PAHs and/or reflect low ISM metallicity. These galaxies also have lower values of $\gamma$ and higher values of $U_{\min }$ than typical spirals.

The dust masses of these early-type galaxies are at least several orders of magnitude lower than the dust masses of spirals. The dust mass of a typical early-type galaxy detected in the far-infrared is $10^{5}-10^{6.5} M_{\odot}$; however, many galaxies are undetected in the long-wavelength MIPS bands and the upper limits on the dust masses in these galaxies range up to an order of magnitude lower, or $10^{4}-10^{5}$. As our sample was designed to be representative of the entire early-type galaxy population, our results indicate that approximately $60 \%$ of typical early-type galaxies have $\geqslant 10^{5} M_{\odot}$ of interstellar dust.

There is no correlation between the presence of dust and galaxy morphology. Our sample contains approximately equal numbers of ellipticals and lenticular galaxies and the same fraction of each morphological type has interstellar dust. We also see no strong evidence for a correlation between the presence of dust and environment. There is a strong correlation between the detection of dust and the presence of visible-wavelength emission lines, which are usually consistent with some form 
of nuclear activity. We detect dust emission from all of the galaxies with nuclear activity, while only approximately $25 \%$ of the galaxies classified as inactive have detectable dust emission. Based on the relatively weak nuclear emission in these galaxies, we conclude that the dust, and the larger reservoir of cold gas associated with it, is a necessary but not sufficient condition for accretion onto the central, supermassive black hole.

We use our dust mass measurements and the demographics of dust in early-type galaxies to demonstrate that most of the dust mass cannot originate from evolved stars. The main argument is that there are order-of-magnitude variations in the dust mass between galaxies with the same morphological type and stellar mass. We also use these data to rule out an exclusively external origin for the dust. An external origin requires that the product of the merger rate of gas-rich galaxies and the dust destruction timescale equals the fraction of dusty early-type galaxies. While many observations of dust lane morphology and gas kinematics provide strong evidence for an external origin, the observed dust masses require mergers of gas-rich galaxies that are at least an order of magnitude too rare to explain the presence of dust in the majority of early-type galaxies. The dust destruction timescale would need to be nearly an order of magnitude greater than the Milky Way value of $4 \times 10^{8} \mathrm{yr}$, rather than the expectation that it is $10^{4}-10^{5} \mathrm{yr}$ due to sputtering in hot gas, in order for purely external accretion to be viable.

We propose instead that the external accretion of cold gas provides an environment for the continual growth of dust for much longer than the nominal destruction timescale of individual grains. If dust grains continue to grow in mass in the externally accreted cold medium, the reduction in the steadystate dust mass should scale with the much longer depletion timescale of the cold gas, rather than the typical destruction timescale for individual grains. In fact, continual dust grain growth in this cold gas seems inevitable, provided the conditions are similar to those that lead to dust grain growth in the Milky Way. The longer gas depletion timescale of several gigayears is consistent with the merger rates of gas-rich satellites, the morphology and kinematics of the cold dust and gas, and the distribution of dust masses. In this scenario, the lifetime for the low-luminosity AGNs present in most dusty, early-type galaxies is also comparable to this several gigayear timescale.

We thank Ramiro Simões Lopes for his assistance with the early stages of this project. We are grateful to Karin Sandstrom and the referee for many comments on the manuscript, as well as to Alison Crocker, Adam Leroy, and Craig Sarazin for helpful discussions. P.M. is grateful for support from the sabbatical visitor program at the North American ALMA Science Center (NAASC) at NRAO and the hospitality of both the NAASC and the University of Virginia while this work was completed. This work is based on archival data obtained with the Spitzer Space Telescope, which is operated by the Jet Propulsion Laboratory, California Institute of Technology, under a contract with NASA. Support for this work was provided by an award issued by JPL/Caltech.

\section{Facilities: HST, Spitzer}

\section{REFERENCES}

Aniano, G., Draine, B. T., Calzetti, D., et al. 2012, ApJ, 756, 138

Athey, A., Bregman, J., Bregman, J., Temi, P., \& Sauvage, M. 2002, ApJ, 571,272

Auld, R., Bianchi, S., Smith, M. W. L., et al. 2013, MNRAS, 428, 1880

Barlow, M. J., Krause, O., Swinyard, B. M., et al. 2010, A\&A, 518, L138

Barlow, M. J., \& Silk, J. 1977, ApJL, 211, L83
Bendo, G. J., Galliano, F., \& Madden, S. C. 2012, MNRAS, 423, 197

Bendo, G. J., Joseph, R. D., Wells, M., et al. 2003, AJ, 125, 2361

Bernard, J. P., Boulanger, F., Desert, F. X., et al. 1994, A\&A, 291, L5

Bertola, F., Bettoni, D., Rusconi, L., \& Sedmak, G. 1984, AJ, 89, 356

Bertola, F., Buson, L. M., \& Zeilinger, W. W. 1992, ApJL, 401, L79

Bregman, J. N., Snider, B. A., Grego, L., \& Cox, C. V. 1998, ApJ, 499, 670

Bressan, A., Granato, G. L., \& Silva, L. 1998, A\&A, 332, 135

Bressan, A., Panuzzo, P., Silva, L., et al. 2007, in ASP Conf. Ser. 374, From Stars to Galaxies: Building the Pieces to Build Up the Universe, ed. A. Vallenari,

R. Tantalo, L. Portinari, \& A. Moretti (San Francisco, CA: ASP), 333

Clemens, M. S., Jones, A. P., Bressan, A., et al. 2010, A\&A, 518, L50

Combes, F., Young, L. M., \& Bureau, M. 2007, MNRAS, 377, 1795

Conroy, C., Gunn, J. E., \& White, M. 2009, ApJ, 699, 486

Cortese, L., Ciesla, L., Boselli, A., et al. 2012, A\&A, 540, 52

Davis, T. A., Alatalo, K., Sarzi, M., et al. 2011, MNRAS, 417, 882

Draine, B. T. 2009, in ASP Conf. Ser. 414, Cosmic Dust: Near and Far, ed. T. Henning, E. Grün, \& J. Steinacker (San Francisco, CA: ASP), 453

Draine, B. T., Dale, D. A., Bendo, G., et al. 2007, ApJ, 663, 866

Draine, B. T., \& Li, A. 2007, ApJ, 657, 810

Draine, B. T., \& Salpeter, E. E. 1979a, ApJ, 231, 438

Draine, B. T., \& Salpeter, E. E. 1979b, ApJ, 231, 77

Dunne, L., Gomez, H. L., da Cunha, E., et al. 2011, MNRAS, 417, 1510

Dwek, E., \& Scalo, J. M. 1980, ApJ, 239, 193

Feigelson, E. D., \& Nelson, P. I. 1985, ApJ, 293, 192

Filho, M. E., Barthel, P. D., \& Ho, L. C. 2006, A\&A, 451, 71

Filho, M. E., Fraternali, F., Markoff, S., et al. 2004, A\&A, 418, 429

Finkbeiner, D. P., Davis, M., \& Schlegel, D. J. 1999, ApJ, 524, 867

Flohic, H. M. L. G., Eracleous, M., Chartas, G., Shields, J. C., \& Moran, E. C. 2006, ApJ, 647, 140

Frayer, D. T., Sanders, D. B., Surace, J. A., et al. 2009, ApJ, 138, 1261

Gomez, H. L., Baes, M., Cortese, L., et al. 2010, A\&A, 518, L45

Gordon, K. D., Galliano, F., Hony, S., et al. 2010, A\&A, 518, L89

Gordon, K. D., Misselt, K. A., Witt, A. N., \& Clayton, G. C. 2001, ApJ, 551,269

Goudfrooij, P., \& de Jong, T. 1995, A\&A, 298, 784

Groves, B., Krause, O., Sandstrom, K., et al. 2012, MNRAS, 426, 892

Helou, G., Roussel, H., Appleton, P., et al. 2004, ApJS, 154, 253

Ho, L. C. 2008, ARA\&A, 46, 475

Ho, L. C., Filippenko, A. V., \& Sargent, W. L. 1995, ApJS, 98, 477

Ho, L. C., Filippenko, A. V., \& Sargent, W. L. W. 1997a, ApJS, 112, 315

Ho, L. C., Filippenko, A. V., Sargent, W. L. W., \& Peng, C. Y. 1997b, ApJS, 112,391

Hubble, E. 1943, ApJ, 97, 112

Hunt, L. K., Giovanardi, C., \& Helou, G. 2002, A\&A, 394, 873

Joblin, C., D'Hendecourt, L., Leger, A., \& Defourneau, D. 1994, A\&A, 281,923

Jones, A. P., Tielens, A. G. G. M., Hollenbach, D. J., \& McKee, C. F. 1994, ApJ, 433, 797

Jura, M., Kim, D. W., Knapp, G. R., \& Guhathakurta, P. 1987, ApJL, 312, L11

Kaneda, H., Onaka, T., \& Sakon, I. 2005, ApJL, 632, L83

Knapp, G. R., Guhathakurta, P., Kim, D.-W., \& Jura, M. A. 1989, ApJS, 70,329

Knapp, G. R., Gunn, J. E., \& Wynn-Williams, C. G. 1992, ApJ, 399, 76

Lauer, T. R., Faber, S. M., Gebhardt, K., et al. 2005, AJ, 129, 2138

Lebzelter, T., Posch, T., Hinkle, K., Wood, P. R., \& Bouwman, J. 2006, ApJL, 653, L145

Leitherer, C., Schaerer, D., Goldader, J. D., et al. 1999, ApJS, 123, 3

Li, W., Chornock, R., Leaman, J., et al. 2011, MNRAS, 412, 1473

Lotz, J. M., Jonsson, P., Cox, T. J., et al. 2011, ApJ, 742, 103

Lutz, D., Sturm, E., Tacconi, L. J., et al. 2008, ApJ, 684, 853

Malhotra, S., Hollenbach, D., Helou, G., et al. 2000, ApJ, 543, 634

Maraston, C. 2005, MNRAS, 362, 799

Mathews, W. G., \& Brighenti, F. 2003, ApJL, 590, L5

Mathis, J. S., Mezger, P. G., \& Panagia, N. 1983, A\&A, 128, 212

Matsuura, M., Dwek, E., Meixner, M., et al. 2011, Sci, 333, 1258

McKee, C. 1989, in IAU Symp. 135, Interstellar Dust, ed. L. J. Allamandola \& A. G. G. M. Tielens (Cambridge: Cambridge Univ. Press), 431

Micelotta, E. R., Jones, A. P., \& Tielens, A. G. G. M. 2010, A\&A, 510, A37 Morganti, R., de Zeeuw, P. T., Oosterloo, T. A., et al. 2006, MNRAS, 371, 157

Nagar, N. M., Falcke, H., \& Wilson, A. S. 2005, A\&A, 435, 521

Piovan, L., Tantalo, R., \& Chiosi, C. 2003, A\&A, 408, 559

Piovan, L., Tantalo, R., \& Chiosi, C. 2006, MNRAS, 370, 1454

Pogge, R. W., \& Martini, P. 2002, ApJ, 569, 624

Popescu, C. C., Misiriotis, A., Kylafis, N. D., Tuffs, R. J., \& Fischera, J. 2000, A\&A, 362, 138 
Ravindranath, S., Ho, L. C., Peng, C. Y., Filippenko, A. V., \& Sargent, W. L. W. 2001, AJ, 122, 653

Rowlands, K., Dunne, L., Maddox, S., et al. 2012, MNRAS, 419, 2545

Sarzi, M., Falcón-Barroso, J., Davies, R. L., et al. 2006, MNRAS, 366, 1151

Sarzi, M., Shields, J. C., Schawinski, K., et al. 2010, MNRAS, 402, 2187

Schutte, W. A., Tielens, A. G. G. M., \& Allamandola, L. J. 1993, ApJ, 415,397

Sellgren, K. 1984, ApJ, 277, 623

Simões Lopes, R. D., Storchi-Bergmann, T., de Fátima Saraiva, M., \& Martini, P. 2007, ApJ, 655, 718

Skibba, R. A., Engelbracht, C. W., Dale, D., et al. 2011, ApJ, 738, 89

Smith, J. D. T., Draine, B. T., Dale, D. A., et al. 2007, ApJ, 656, 770

Smith, M. W. L., Gomez, H. L., Eales, S. A., et al. 2012, ApJ, 748, 123

Stewart, K. R., Bullock, J. S., Barton, E. J., \& Wechsler, R. H. 2009, ApJ, 702,1005
Suh, K.-W. 2002, MNRAS, 332, 513

Sun, M., Vikhlinin, A., Forman, W., Jones, C., \& Murray, S. S. 2005, ApJ, 619,169

Temi, P., Brighenti, F., Mathews, W. G., \& Bregman, J. D. 2004, ApJS, 151,237

Temi, P., Brighenti, F., \& Mathews, W. G. 2007, ApJ, 660, 1215

Temi, P., Brighenti, F., \& Mathews, W. G. 2009, ApJ, 707, 890

Tran, H. D., Tsvetanov, Z., Ford, H. C., et al. 2001, AJ, 121, 2928

Tsai, J. C., \& Mathews, W. G. 1995, ApJ, 448, 84

van Dokkum, P. G., \& Franx, M. 1995, AJ, 110, 2027

Verdoes Kleijn, G. A., \& de Zeeuw, P. T. 2005, A\&A, 435, 43

Welch, G. A., Sage, L. J., \& Young, L. M. 2010, ApJ, 725, 100

Xilouris, E. M., Madden, S. C., Galliano, F., Vigroux, L., \& Sauvage, M. 2004, A\&A, 416, 41

Young, L. M., Bureau, M., Davis, T. A., et al. 2011, MNRAS, 414, 940 